This is an Open Access article, distributed under the terms of the Creative Commons Attribution licence (http://creativecommons.org/licenses/by/4.0/), which permits unrestricted re-use, distribution, and reproduction in any medium, provided the original work is properly cited.

doi:10.1017/jfm.2015.335

\title{
Antibubbles and fine cylindrical sheets of air
}

\author{
D. Beilharz ${ }^{1}$, A. Guyon ${ }^{1}$, E. Q. Li ${ }^{2} \dagger$, M.-J. Thoraval ${ }^{2,3}$ and \\ S. T. Thoroddsen ${ }^{2} \uparrow$ \\ ${ }^{1}$ Département de Mécanique, Ecole Polytechnique, 91128 Palaiseau CEDEX, France \\ ${ }^{2}$ Division of Physical Sciences and Engineering and Clean Combustion Research Center, \\ King Abdullah University of Science and Technology (KAUST), Thuwal 23955-6900, Saudi Arabia \\ ${ }^{3}$ Physics of Fluids Group, Faculty of Science and Technology, MESA+ Institute, University of Twente, \\ 7500 AE Enschede, The Netherlands
}

(Received 11 October 2014; revised 31 May 2015; accepted 15 June 2015; first published online 14 August 2015)

Drops impacting at low velocities onto a pool surface can stretch out thin hemispherical sheets of air between the drop and the pool. These air sheets can remain intact until they reach submicron thicknesses, at which point they rupture to form a myriad of microbubbles. By impacting a higher-viscosity drop onto a lower-viscosity pool, we have explored new geometries of such air films. In this way we are able to maintain stable air layers which can wrap around the entire drop to form repeatable antibubbles, i.e. spherical air layers bounded by inner and outer liquid masses. Furthermore, for the most viscous drops they enter the pool trailing a viscous thread reaching all the way to the pinch-off nozzle. The air sheet can also wrap around this thread and remain stable over an extended period of time to form a cylindrical air sheet. We study the parameter regime where these structures appear and their subsequent breakup. The stability of these thin cylindrical air sheets is inconsistent with inviscid stability theory, suggesting stabilization by lubrication forces within the submicron air layer. We use interferometry to measure the air-layer thickness versus depth along the cylindrical air sheet and around the drop. The air film is thickest above the equator of the drop, but thinner below the drop and up along the air cylinder. Based on microbubble volumes, the thickness of the cylindrical air layer becomes less than $100 \mathrm{~nm}$ before it ruptures.

Key words: breakup/coalescence, bubble dynamics, drops and bubbles

\section{Introduction}

When a drop impacts on a pool surface at low velocity, it can bounce from the surface (Couder et al. 2005; Molacek \& Bush 2013), entrap a bubble under its centre (Thoroddsen, Etoh \& Takehara 2003; Liow \& Cole 2007) or stretch out a hemispherical layer of air which then breaks up into a multitude of microbubbles,

$†$ Email addresses for correspondence: Erqiang.Li@KAUST.edu.sa, sigurdur.thoroddsen@kaust.edu.sa 
frequently named Mesler entrainment (Sigler \& Mesler 1990). Mills, Saylor \& Testik (2012) showed that the breakup of this air layer is inherently random for water, while Saylor \& Bounds (2012) demonstrated that the thin air films are much more stable for some other liquids, such as silicone oils and ethanol (Sundberg-Anderson \& Saylor 2014). This repeatability allowed Thoroddsen et al. (2012) to study details of the air-film breakup, employing triggered imaging with an ultra-high-speed CCD video camera (Etoh, Poggemann \& Kreider 2003). They explained the formation of various microbubble morphologies, from bubble necklaces to chandeliers, as well as estimating the air-film thickness from the volume of microbubbles left behind when it ruptures. Subsequent work by Tran et al. (2013) used colour interferometry to measure the air-film thickness, but only under the centre of the drop.

In the present study we observe these air films for silicone oils when we increase the viscosity of the drop liquid while keeping the pool liquid at low viscosity. This slows down the shape deformations of the drop and allows the formation of thin air layers with new geometries, which cannot be realized when the drop and pool are of the same viscosity. Specifically, we focus on the formation and breakup of thin cylindrical sheets of air, as well as repeatable antibubbles.

For surfactant-laden liquids, such as beer, antibubbles are readily generated by jet or drop impacts onto a pool surface, see e.g. Dorbolo et al. $(2005,2010)$ and Kim \& Stone (2008). Vandewalle et al. (2009) used this technique to generate composite emulsions. Scheid et al. (2012) and Scheid, Zawala \& Dorbolo (2014) have studied how the gas drainage and dissolution affect the lifetime of the surfactant-stabilized antibubbles. In the present work no surfactants are present to stabilize any of the free surfaces.

\section{Experimental set-up}

\subsection{Liquids, nozzle, drops and threads}

The experimental configuration consists of the impact of a viscous drop onto a less viscous pool liquid. We use a wide range of miscible silicone oils, with the pool liquid viscosity $v_{p}$ ranging mostly between 0.65 and $10 \mathrm{cSt}$, while the drop viscosity $v_{d}$ is varied from 5 to $10000 \mathrm{cSt}$. A limited set of experiments were conducted with higher viscosities in the pool, up to $v_{p}=100$ and $350 \mathrm{cSt}$, but these impacts demanded more frequent changes of pool liquid, reducing the number of realizations. Table 1 lists the properties of the various silicone oils and their suppliers.

Figure 1 shows a schematic of the experimental set-up. The pool is held inside a rectangular-cross-section glass container of approximately $4 \mathrm{~cm} \times 5 \mathrm{~cm}$ inner crosssection and $5 \mathrm{~cm}$ depth.

The drops were in most cases pinched off from a metallic nozzle of $1.6 \mathrm{~mm}$ outer diameter, except in $\S 4$, where we study the effect of drop size on the phase diagram. The drop liquids wet the metallic nozzle and are therefore released from its outer diameter, which for the $1.6 \mathrm{~mm}$ nozzle gives drop diameters $D \simeq 2.4 \mathrm{~mm}$. The drops were selected to have larger density and higher viscosity than the pool liquid. This makes them sink to the bottom of the tank and reduces the contamination of the pool liquid. As the drop and pool liquids were miscible, care was taken to verify that the overall dynamics did not change after numerous drop impacts had been performed. This was done by comparing with experiments using fresh liquid in the pool. This showed that a large number of realizations can be performed without any observable effects.

For the larger viscosities the flow rate through the nozzle can have a significant effect on the released drop shape, as viscous stresses modify the usual balance 


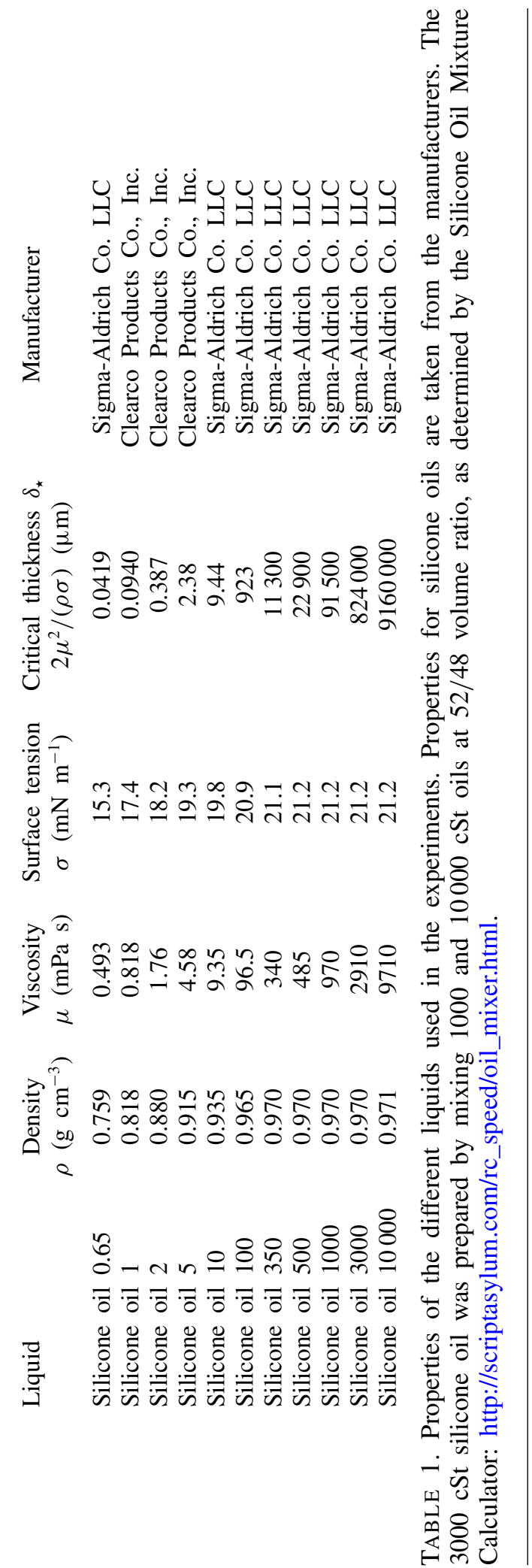


(a)

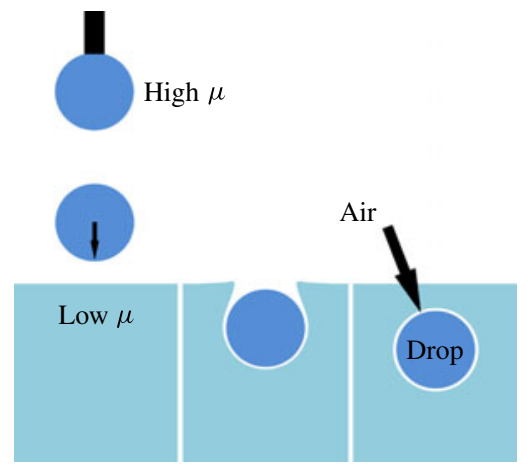

(b)

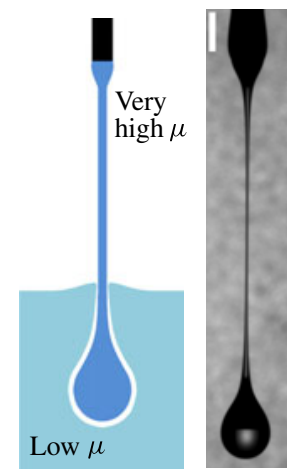

FIGURE 1. (Colour online) Sketch of the experimental configuration, showing the nozzle used to pinch off the higher-viscosity drop, to impact onto a lower-viscosity pool. (a) The drop can form an antibubble when the air layer wraps around the entire drop. (b) For very large drop viscosities a thread of drop liquid connects to the nozzle leading to the formation of an extended cylinder of air between the drop and the pool. Image of the pinch-off of a drop from a nozzle in air, for a viscosity of $500 \mathrm{cSt}$, with a $2 \mathrm{~mm}$ scale bar.

between gravity and surface tension. We therefore used a syringe pump to feed the liquid at a slow $\left(10 \mu \mathrm{l} \mathrm{min}{ }^{-1}\right)$ and repeatable rate into the drop. A separate set of experiments measured the fall velocity of the drops $U$, as the viscous stress in the thread slows them down, below what is expected from free-fall calculations, based on $U=\sqrt{2 g H}$, where $g$ is gravity and $H$ is the release height. For the highest drop viscosities the pinch-off pulls out a thread which does not detach from the nozzle during the impact. The image in figure $1(b)$ shows such a case.

\subsection{Imaging and parameter range}

The droplets were imaged using long-distance microscopes, either adjustable magnification Leica Z16 APO, or fixed Mitutoyo objectives between 5 and $50 \times$, giving a corresponding pixel resolution of between 1.6 and $31.5 \mu \mathrm{m}$. The rapid film rupture requires high-speed video at frame rates of up to $300 \mathrm{kfps}$, which were acquired with a Phantom V1610-CMOS video camera. Back-lighting used a $350 \mathrm{~W}$ metal-halide lamp (Sumita), which was shone onto a diffuser. For more details see Li et al. (2014a).

The impact height was always below $50 \mathrm{~mm}$. The interesting phenomena focused on herein occur for Reynolds and Weber numbers based on the pool properties, in the range

$$
R e=\frac{\rho_{p} U D}{\mu_{p}}<2400, \quad W e=\frac{\rho_{p} D U^{2}}{\sigma}<100,
$$

where $D$ and $U$ are the drop diameter and impact velocity; $\rho_{p}, \mu_{p}$ are the density and dynamic viscosity of the pool and $\sigma$ is the surface tension between the air and the pool. The surface tension of the more viscous drop liquid (table 1) is typically slightly larger than that of the pool liquid, but the two liquids are always miscible. The much larger value of $R e$ than We suggests that the dynamics of the cavity inside the pool is more influenced by surface tension than viscosity. 


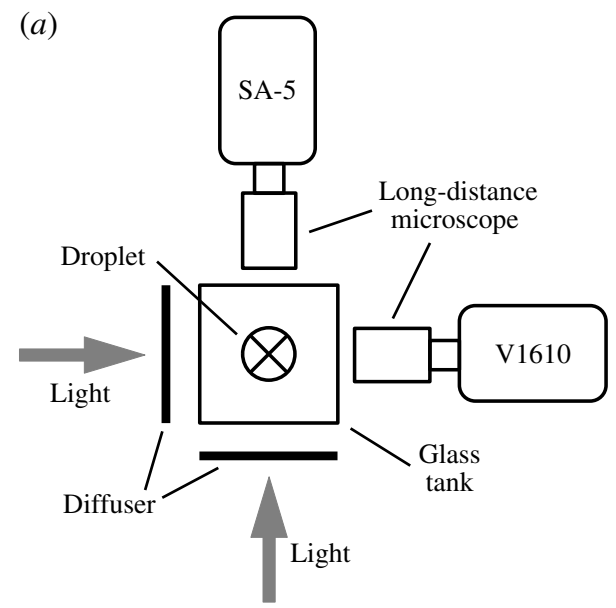

(b)

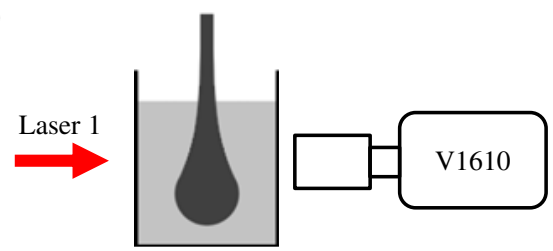

(c)

(d)

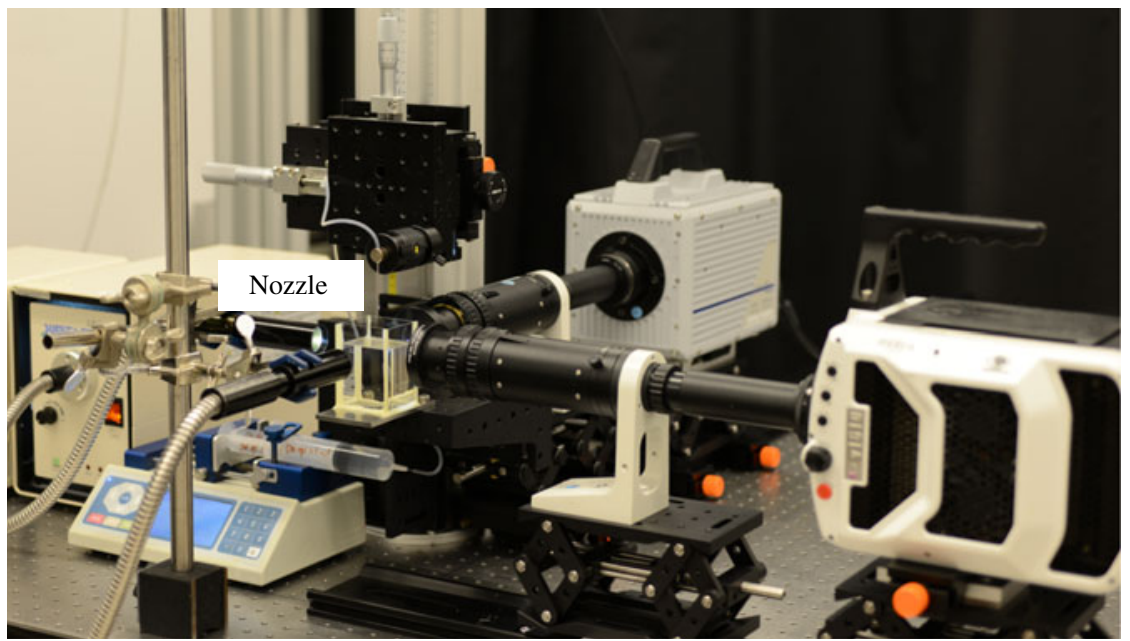

FIGURE 2. (Colour online) Sketches of the experimental set-ups. (a) Two-camera views, used to study the fine details of the breakup of the air cylinder. Set-up for interferometry, using back-lighting $(b)$ and front-lighting with a beam splitter $(c)$. (d) Photograph of the set-up with two high-speed cameras (Phantom V1610 and Photron SA-5) and showing the long-distance microscopes, two metal-halide lamps, the glass container, a syringe pump to feed the drop through the nozzle and micrometers to change the release height.

\subsection{Two perpendicular camera views}

During the breakup of the fine cylindrical sheet of air, the axisymmetry is lost and in order to accurately measure the microbubble sizes we need two perpendicular views of the process, as is shown in figure 2. To accomplish this we used two high-speed video cameras, i.e. Phantom V1610 and Photron SA-5, which were synchronized with the same external trigger. In this way we guaranteed that all of the microbubbles were identified and errors in estimating their volume were minimized. Such errors can arise from the limited depth of focus and the refractive index difference between the two liquids, which can cause slight distortions between microbubbles sitting in front of or behind the viscous thread. Both cameras used the same Leica long-distance 
microscopes listed above, but the difference in the pixel sizes of the two sensors results in slightly different magnifications for the two views. Pixel resolution in the range $1-2 \mu \mathrm{m}$ was used.

\subsection{Interference imaging}

Some of the imaging was conducted with monochromatic light to allow for interference imaging, to directly measure the thickness of the air films. Here we used solid-state pulsed diode lasers (SI-LUX640 from Specialised Imaging, Tring, UK), which produce red light of wavelength $\lambda=640 \mathrm{~nm}$. The variation in air-layer thickness between the centres of a bright and a dark fringe is therefore $\lambda / 4=160 \mathrm{~nm}$. However, the free surface is not perfectly vertical and slopes at angles as large as $\theta=26^{\circ}$. This affects the measurements through Snell's law of refraction, as the index of refraction of the silicone oil is 1.35 . Therefore, the light travels a longer distance through the air layer before interfering, thereby overestimating its thickness. This is corrected for, based on the measured local slope of the free surface (see figure 17). However, the slope at the bottom of the drop, in the side view, is too large to apply this technique there.

We used a laser pulse duration of $10 \mathrm{~ns}$, synchronized with the video frames of the high-speed camera, using frame rates of up to $30 \mathrm{kfps}$. This allowed us to follow the motion of individual fringes in time. To measure absolute thicknesses we therefore simply need a reference fringe, which is given by the thinnest section just before film rupture.

Care was taken to verify that we were observing interference arising from only one of the air layers. This was done in numerous ways: by shifting the focal plane of the microscope, changing the aperture, imaging with and without a diffuser, as well as changing the orientation of the lighting, as shown in figure $2(b, c)$. Supplementary figure S4 available at http://dx.doi.org/10.1017/jfm.2015.335 compares the fringes for back- and front-lighting, showing no significant changes in the pattern. Finally, we observed the interference fringes during the zipper breakup of the air film, where the film breaks along the azimuthal direction and thereby for a fleeting moment only one air film is present. In all cases, we did not see any overall changes in the interference pattern, showing conclusively that we were observing interference from only one of the air layers.

\section{Results}

\subsection{Overall parameter regimes}

While we have extended the parameter regime studied by Thoroddsen et al. (2012) to include different viscosities of the drop and pool liquids, we have observed many of the same regimes as described in that earlier paper. However, our focus is here on new phenomena that appear, especially when the drop viscosity is much larger than that of the pool. For reference, we start by showing some of the main previously described phenomena in figure 3 . These include rebounding from the pool surface (figure $3 a$ ), when all the kinetic energy of the drop is overcome by the surface energy of the deformed crater within the pool. The surface goes through large deformation, but the net curvature of the neck is either positive or the more viscous inner liquid cylinder can here prevent pinch-off, by cushioning through the air layer, thus allowing surface tension to pull the drop back up. Rebounding can therefore occur for larger penetrations than were observed in Thoroddsen et al. (2012). The details of the rebound differ somewhat from one realization to the next due to the compression and 
(a)

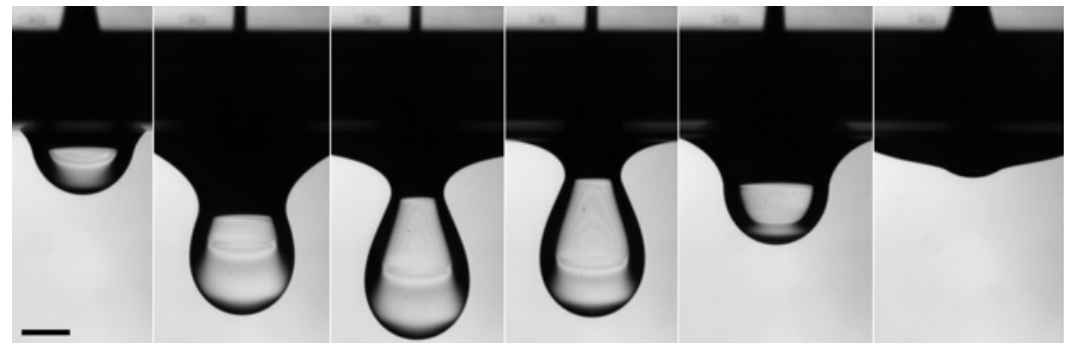

(b)

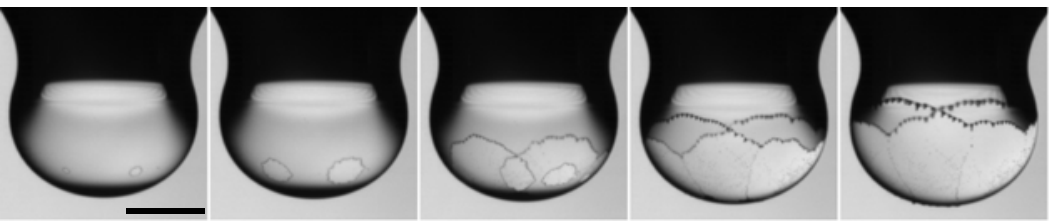

(c)

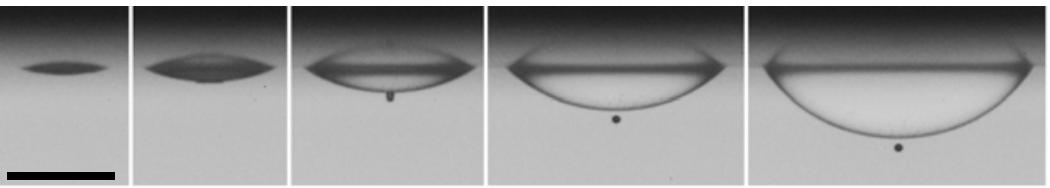

FIGURE 3. Three typical phenomena during droplet-pool interaction. (a) Large surface deformations during the rebound of a drop, for $v_{d}=10000 \mathrm{cSt}$ and $v_{p}=10 \mathrm{cSt}$, from $H=27.5 \mathrm{~mm}$. The times shown are $t=3.3,14.3,25.3,39.5,53.6$ and $61.1 \mathrm{~ms}$ after the drop reaches the pool surface. (b) Mesler breakup of the hemispheric air film, for $v_{d}=500 \mathrm{cSt}$ and $v_{p}=10 \mathrm{cSt}$, from $H=22.0 \mathrm{~mm}$. (c) Early entrapment of a central disc of air, which quickly contracts into a bubble, for $v_{d}=3000 \mathrm{cSt}$ and $v_{p}=1 \mathrm{cSt}$, from $H=90 \mathrm{~mm}$. The scale bars are $1 \mathrm{~mm}$.

buckling of the viscous thread, as shown in the supplementary material. Buoyancy plays no role in the rebounding, as the density of the drop is always larger than that of the pool and the volume of the air sheath is minimal. For slightly larger impact velocities (figure $3 b$ ), the air hemisphere ruptures at numerous holes, which grow in size and leave streams of microbubbles where they meet. This is the so-called Mesler entrainment (Sigler \& Mesler 1990; Mills et al. 2012; Thoroddsen et al. 2012). For still larger impact velocity, we show, in figure 3(c), the early breakup of the air layer which entraps a central air disc, a problem extensively studied for higher impact velocities (Thoroddsen et al. 2003).

We next present the overall shape of the phase space of phenomena observed over a range of drop viscosities $\mu_{d}$, expressed by the Ohnesorge number based on drop properties,

$$
O h_{d}=\frac{\mu_{d}}{\sqrt{\rho_{d} R \sigma}}
$$

and impact heights in terms of $W e_{p}$ based on pool properties. Here, $R=D / 2$ is the drop radius. The pool viscosity was also varied, but a majority of the experiments were performed with the lowest values of $v_{p}=0.65$ and $1.0 \mathrm{cSt}$.

Figure 4 includes the various previously observed regimes, like rebounding drops and Mesler ruptures forming necklaces or chandeliers of microbubbles. We also highlight with larger symbols ( $\bullet$, green online; 4$)$ the regions where antibubbles and 

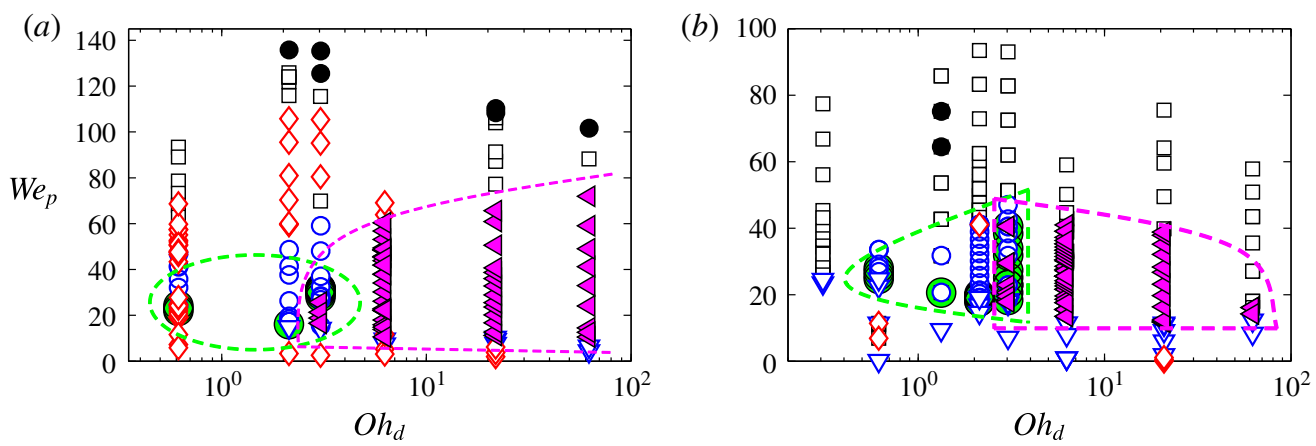

FIGURE 4. Overview of observed phenomena in the different parameter regimes, when the drop liquid viscosity is varied while the pool liquid is $(a) 0.65 \mathrm{cSt}$ and $(b) 1.0 \mathrm{cSt}$. Antibubbles are indicated by (๑, green) and air cylinders by (४); ( $\square$ ) indicates Mesler breakup of the film and $(\diamond)$ indicates zipper breakup; $(\nabla)$ shows bouncing of the drop from the free surface; (৩) indicates early entrapment of an air disc and central bubble; (O) are antibubbles that fail to fully close. The dashed curves give approximate outlines of the regimes where antibubbles (green) and cylindrical air sheets (pink) occur.

cylindrical sheets of air are formed respectively. It should be noted that we do not suggest that these plots are universal regime diagrams, as we have only used one nozzle size. Rather, the $O h_{d}$ is a convenient way of non-dimensionalizing the drop viscosity, which is the physical parameter we are changing along the $x$-axis. The Bond number of the nozzle will certainly affect the drop shape during the pinch-off, as will be shown in $\S 4$ for different nozzle sizes. In other words, separate processes control the pinch-off of the drop from the nozzle and its dynamical interactions with the pool.

Figure 5 shows the regimes for increased pool viscosities. It shows antibubbles forming for pool viscosity only up to $2 \mathrm{cSt}$, whereas cylindrical air films are observed for $v_{p}$ up to $5 \mathrm{cSt}$.

\subsection{Film rupture speeds for Mesler breakup}

We observe large regions of parameter space where Mesler entrainment occurs, i.e. breakup of the thin hemispheric films under the drop into a myriad of microbubbles. The rupture speed of the thin air films is principally determined by two factors, i.e. the thickness of the air film and the viscosity of the surrounding liquid (Reyssat \& Quéré 2006; Thoroddsen et al. 2012). Figure 6 demonstrates that this velocity is principally determined by the lower-viscosity liquid, which is here always the pool viscosity $v_{p}$. The data show a large spread for each drop viscosity $v_{d}$, with the error bars showing the variance away from the mean values. This is because the figure is a conglomeration of the different impact velocities and thereby represents a multitude of different Mesler breakup mechanisms and air-layer thicknesses $\delta$. However, for the most complete data sets presented here, i.e. for $v_{p}=1$ and $10 \mathrm{cSt}$, it is clear that there is only a slight reduction in rupture speed as the drop viscosity $v_{d}$ is increased. For $v_{p}=1 \mathrm{cSt}$ the rupture velocity is only reduced by approximately $33 \%$ when $v_{d}$ is increased by a factor of 2000. We conclude that the much larger drop viscosity plays a minor role in the speed of the air-layer rupture. It should be kept in mind that here the two liquids are miscible and different dynamics can occur for immiscible liquids, where the triple line provides extra resistance (Li et al. $2014 a)$. 


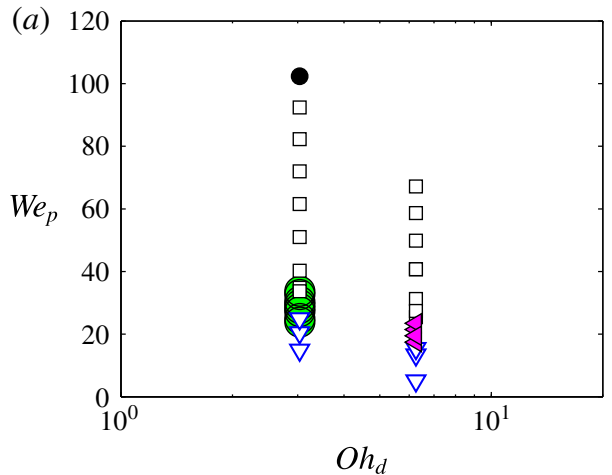

(b)
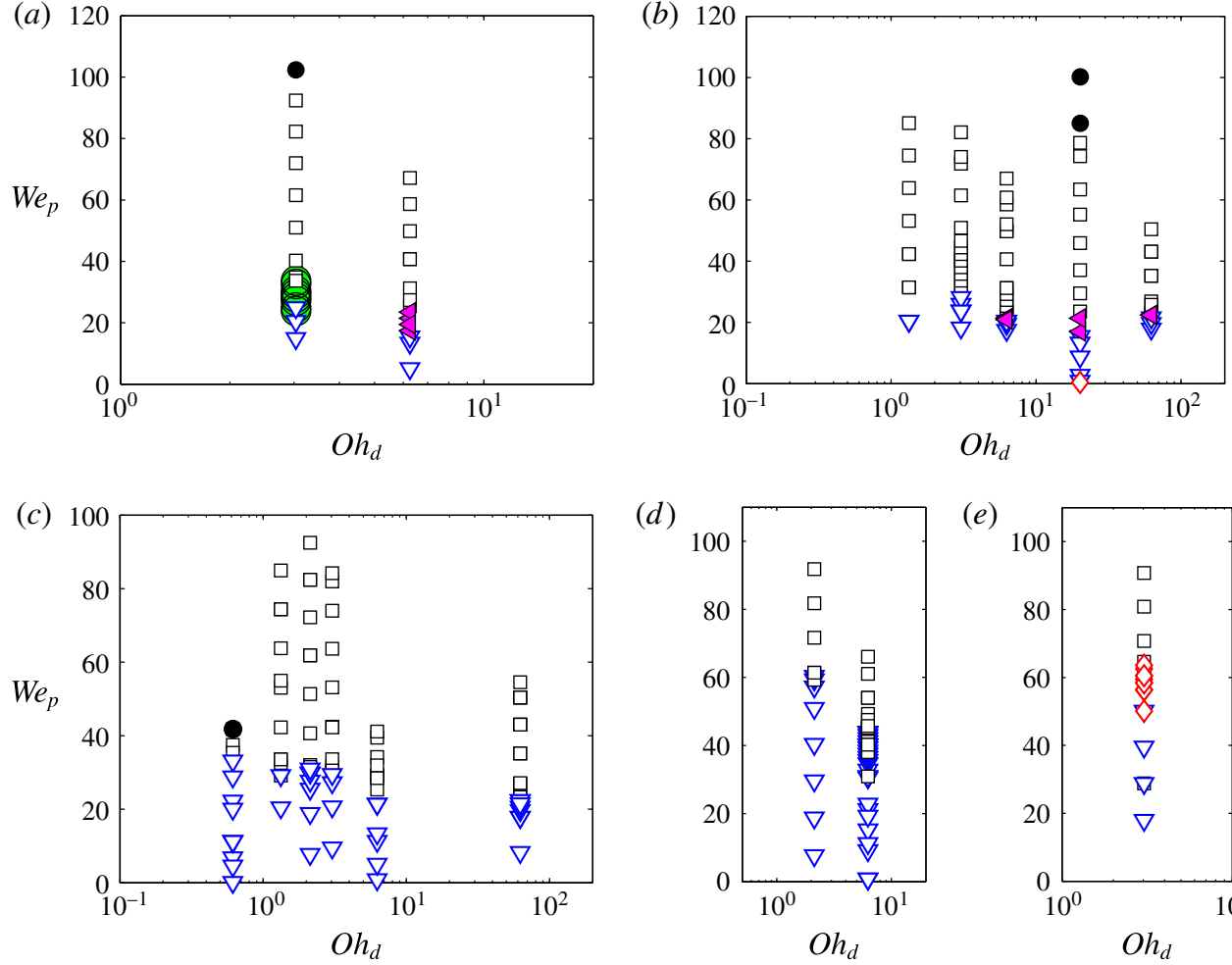

(d)

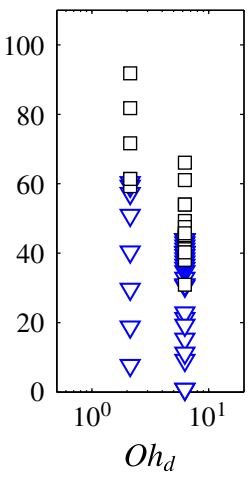

(e)

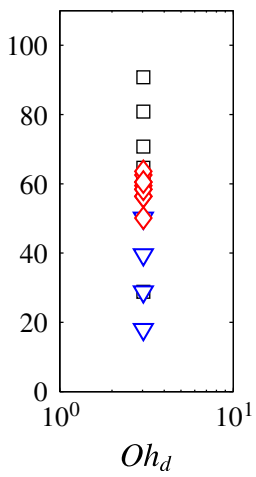

FIGURE 5. (Colour online) Overview of the observed phenomena in the different parameter regimes, when the drop liquid viscosity is varied while the pool liquid is (a) $2.0 \mathrm{cSt}$, (b) $5.0 \mathrm{cSt}$, (c) $10 \mathrm{cSt},(d) 100 \mathrm{cSt}$ and (e) $350 \mathrm{cSt}$. The symbols are the same as in the previous figure.

\subsection{Zipper breakup}

Figure 7 shows what we call zipper breakup, where the rupture moves rapidly in the azimuthal direction around the drop. We have observed this type of breakup more prominently here than in the previous work of Thoroddsen et al. (2012). This type of rupture moves along an azimuthal ring of thinner air film and its speed is faster than the edge rupture in the surrounding film, as is clear by the angle of the surrounding rupture. This often occurs at the upper limit of the film, but sometimes these rings are present above the equator of the drop and rupture when the drop has penetrated much deeper, as in figures $10(b)$ and 21 . The thinning near the surface is measured and discussed in $\$ 3.7$, but the localized thinner rings at the deeper locations can be traced back to the original enwrapping at the pool surface, as discussed in $\S 4$. Figure $7(c)$ also shows a case when there are two azimuthal rings, which rupture at the same time.

In all descending cases the bubble surface remains smooth until rupture. Only during the rebounding of the bottom hemisphere do we in isolated conditions observe surface texture akin to an orange peel, shown in figure 8, just before the bottom rupture occurs. Here, the characteristic length scale of the pattern is approximately $50 \mu \mathrm{m}$. The rebounding can laterally compress the air film, which could lead to an instability. However, we cannot determine which of the two interfaces forms the undulations, and the nature of this instability is thereby unclear and needs further study. 


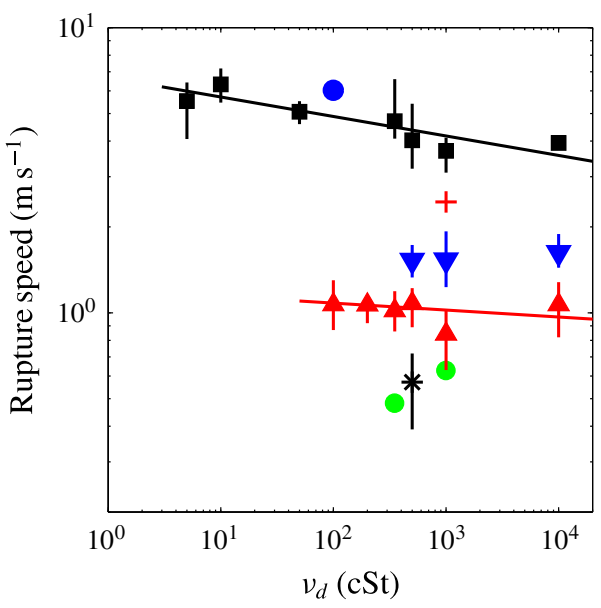

FIGURE 6. The hole rupture speed versus the viscosity of the drop $v_{d}$, for a range of different pool viscosities $v_{p}=0.65 \mathrm{cSt}(\bullet$, blue $) ; 1.0 \mathrm{cSt}(\boldsymbol{\nabla}) ; 2 \mathrm{cSt}(+) ; 5 \mathrm{cSt}(\boldsymbol{\nabla}) ; 10 \mathrm{cSt}$ $(\mathbf{\Delta}) ; 100 \mathrm{cSt}(\bullet$, green) and $350 \mathrm{cSt}(*)$.

\subsection{Formation of antibubbles}

Drop impacts can form antibubbles, e.g. see Dorbolo et al. $(2005,2010)$ and Kim \& Stone (2008). In a limited parameter regime in figures 4 and 5, we observe repeatable formation of such antibubbles, here without the support of surfactants used in these previous studies. For the pinch-off of the air cylinder to successfully close up the air sheet to form an antibubble, the liquid thread must break first before the air cylinder closes up. This excludes antibubble formation for the highest-viscosity drops and leads to the formation of extended air cylinders, as shown in the next subsection. However, this does not exclude the possibility of antibubble formation, if spherical drops were produced in a different way, rather than pinching them off from a nozzle. Figure 9(a) shows a typical sequence of antibubble formation and breakup (see also the supplementary video). Here, the air film remains intact for $129 \mathrm{~ms}$ after the start of its formation. Then it breaks from the bottom of the antibubble (arrow in sixth panel). Based on the volume of the remaining air bubble, the average air thickness is here $\bar{\delta} \simeq 2.2 \mu \mathrm{m}$, with a much thinner layer on the bottom hemisphere where it first ruptures by van der Waals forces. Figure $9(b)$ follows the antibubble and points out interference patterns moving up along the surface, indicating thinning of the air layer along the bottom.

It should be noted that when the thicker rim of the air layer converges on top of the drop, it entraps a small droplet, which bounces around inside the small bubble (figure $9 c$ and supplementary video). The basic mechanism for this entrapment is that the added mass of liquid has vertical momentum, due to the curved surface, thereby pulling the air rim upwards and forcing closure above the horizontal top.

The antibubbles were observed for $v_{p}$ of $0.65,1$ and $2 \mathrm{cSt}$. The air-film dynamics and breakup are quite repeatable from one realization to the next, as is demonstrated in the supplemental material. For example, the time duration from first impact to breakup varies by only $3 \%$ between three subsequent realizations. The interference patterns in figure $9(b)$ are also repeatable. 
(a)

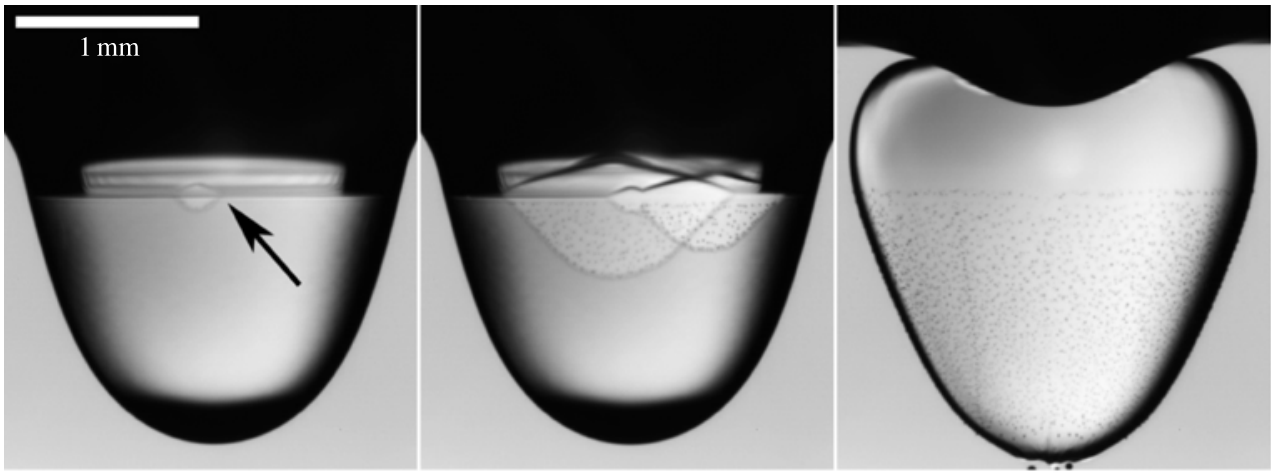

(b)
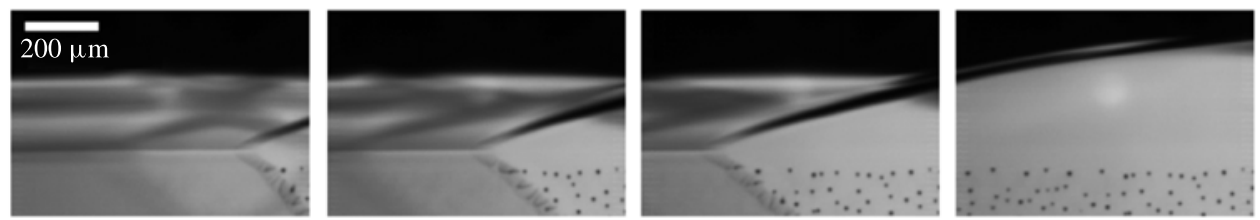

(c)

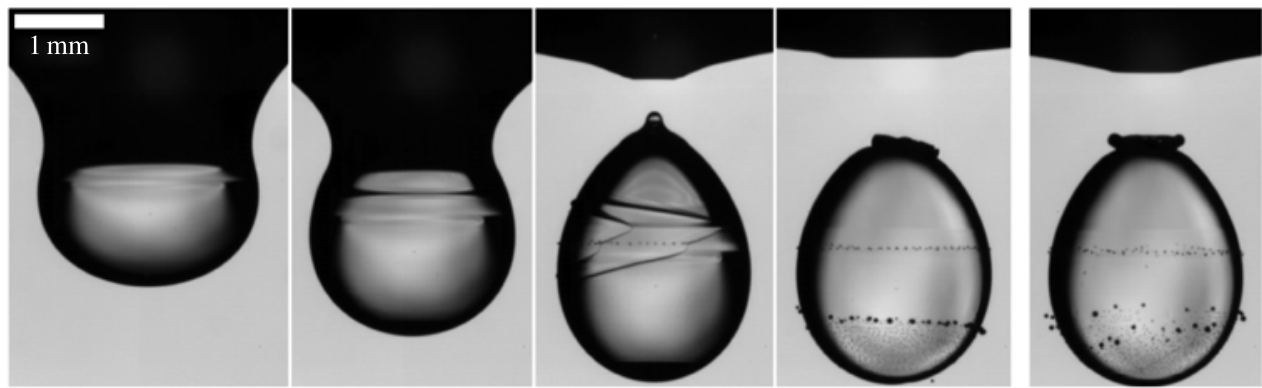

FIgURE 7. Zipper rupture, for $v_{d}=10 \mathrm{cSt}$ and $v_{p}=1 \mathrm{cSt}, H=10 \mathrm{~mm}$. (a) The frames are 17.129, 17.208 and $22.040 \mathrm{~ms}$ after the first deformation of the pool by the drop. $(b)$ The close-up frames (from a different realization) are spaced by $30 \mu \mathrm{s}$. The tip of the rupture moves at $8.2 \mathrm{~m} \mathrm{~s}^{-1}$. (c) The double zipper leaves a line of bubbles in between the two lines. The last frame shows microbubbles from a different realization.

\subsection{Cylindrical air films}

When the pinch-off thread is still connected to the nozzle during the wrapping of the sheet around the drop, instead of an antibubble we observe the formation of cylindrical sheets of air. This is observed for some large-viscosity drops, impacting on $0.65,1.0,2.0$ and $5.0 \mathrm{cSt}$ viscosity pools. Figure 14 shows the slowdown of the bottom of the drop as it travels further into the pool.

Figures 10-12 show some of the various rupture mechanisms observed. In figure 10(a) the film ruptures both near the bottom and near the free surface, leaving a ring of bubbles where the two rupture fronts meet. In figure 11 the thread ruptures close to the free surface and propagates downwards, while in figure $10(b)$ it ruptures along a thinner ring, in a zipper fashion. The initial speed of the air front in figure $11(a)$ is approximately $7 \mathrm{~m} \mathrm{~s}^{-1}$. The diameter of the viscous thread during this rupture is $100 \mu \mathrm{m}$ at the free surface, growing to $136 \mu \mathrm{m}$ at the bottom of the image. 


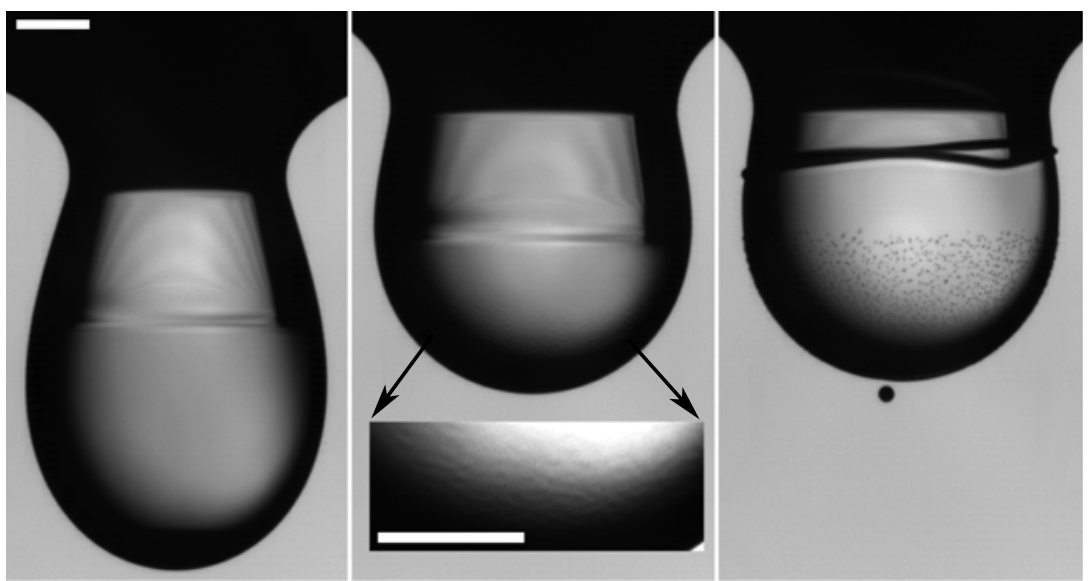

FIGURE 8. The bottom section of the retracting air layer develops the texture of orange peel, just before it ruptures, for $v_{d}=50 \mathrm{cSt}, v_{p}=1 \mathrm{cSt}, H=16 \mathrm{~mm}$. The frames are 14.9 and $15.6 \mathrm{~ms}$ after the maximum depth is reached in the first frame. The scales are $500 \mu \mathrm{m}$.

It should be kept in mind that the conical/trumpet shape of the thread will generate a net capillary pressure gradient away from its thinnest section, due to the gradient in the total curvature of the outer air surface. This is expressed by the Young-Laplace law; see the similar situation for a drop climbing on a cone in Lorenceau \& Quéré (2004) and Li \& Thoroddsen (2013). The air is thereby driven down along the thread, except close to the pool surface where the air can escape upwards. There is therefore an intermediate region near to the top where air is drained away from a ring that thins, thereby facilitating rupture, as shown in figures 11, 13, 16 and 18. A similar situation arises during the drainage of a soap bubble at a free surface (Lhuissier \& Villermaux 2012).

\subsection{Overall thickness of the air layer and capillary pressure}

Figure 12 shows a realization where the extended air layer punctures at the bottom and unravels all the way to the pool surface. The speed of the edge of the rupture (figure 12c) reveals information about how the layer thickness varies along the vertical direction. The air layer is thinnest under the drop, where the initial impact has squeezed out and stretched the layer. This is clear in the distribution of minute bubbles when the air layer ruptures and by the speed of the rupture front. The finest bubbles are below the equator of the drop. On the top hemisphere of the drop and half-way up along the thread, the air layer is much thicker and the layer unwraps without leaving bubbles, thereby forming a torus of air which moves up the thread (see figure 12a, panel 4). This torus becomes unstable to azimuthal capillary instability, breaking from one side and contracting into a bubble at the opposite side of the thread. The air layer continues to rupture above this location travelling up along the thinner cylinder leaving microbubbles. We can estimate the average layer thickness $\bar{\delta}$ in the intermediate region, where no microbubbles are shed and all the air volume collects into a large bubble (last panel). Using the axisymmetric area from the equator towards the rupture above the bubble, we obtain $\bar{\delta}=2.9 \mu \mathrm{m}$. This is the thickest part of the sheet, as is clear from the slowest tangential velocity of the edge, 
(a)

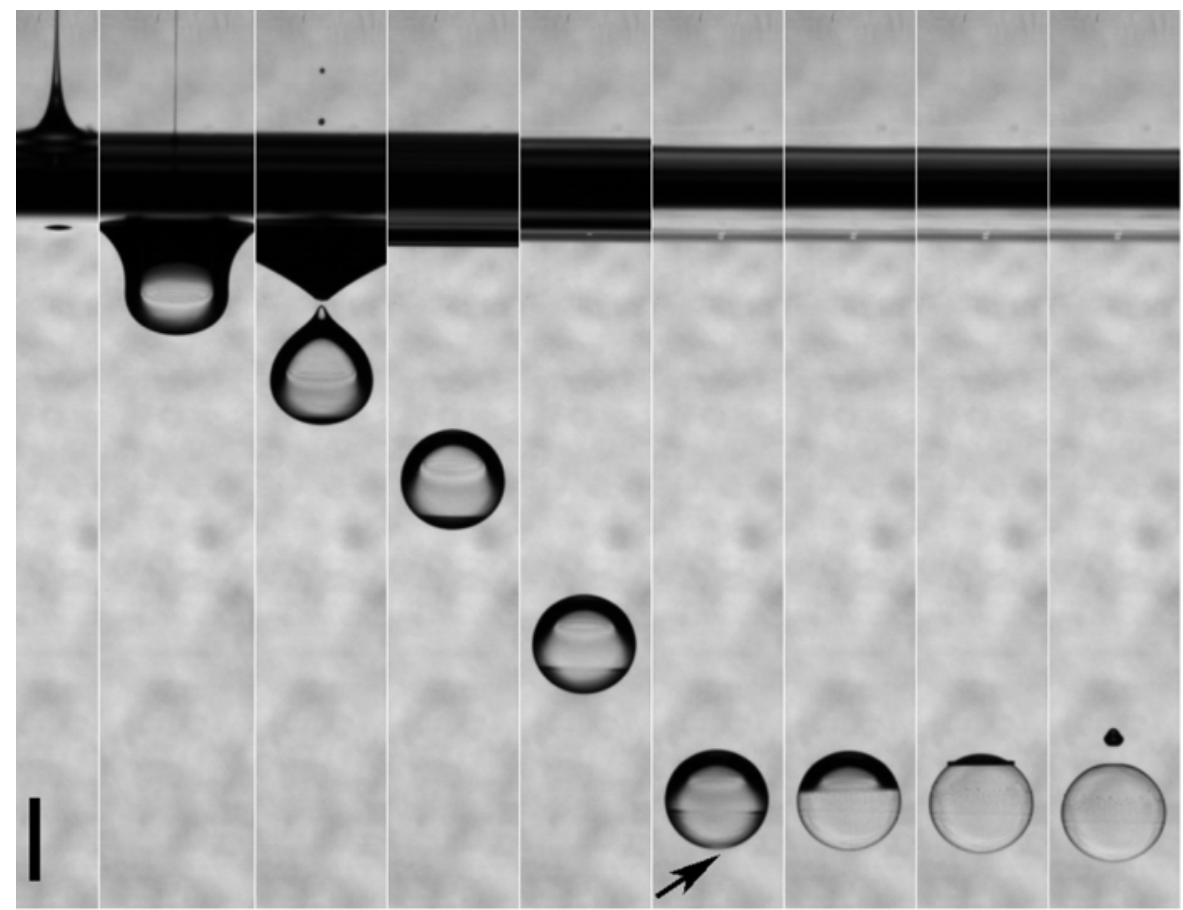

(b)

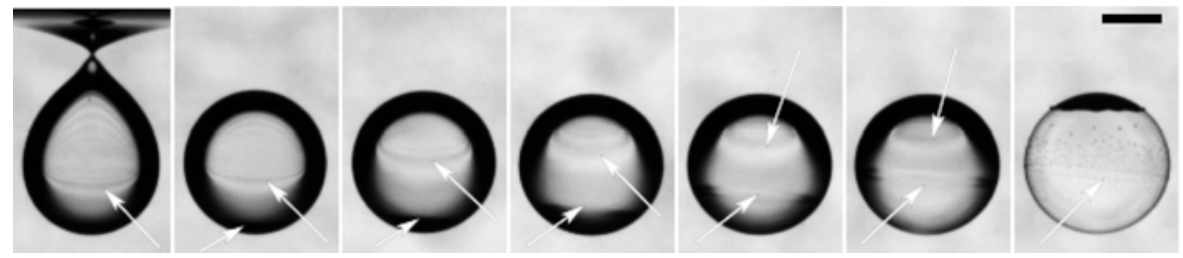

$(c)$

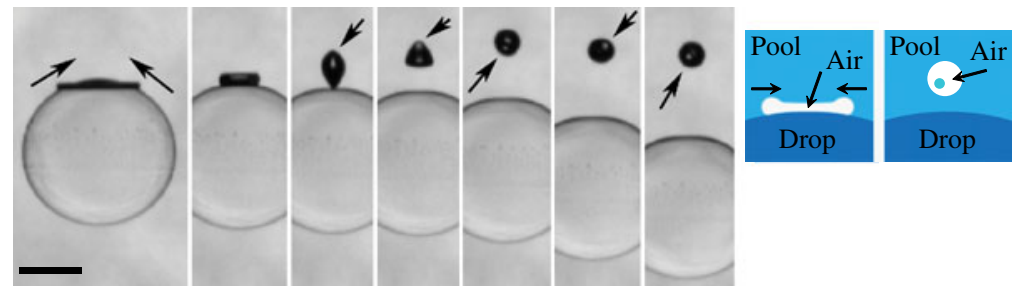

FIgURE 9. (Colour online) (a) Formation and breakup of an antibubble, for $v_{d}=500 \mathrm{cSt}$ and $v_{p}=1 \mathrm{cSt}$. The impact height is $H=19 \mathrm{~mm}$. The frames are shown at $t=$ $-18.6,-11.3,0,23.6,67.1,110.7,111.1,111.9$ and $114.4 \mathrm{~ms}$ relative to the pinch-off of the air cylinder in the third panel. Notice that the trailing thread breaks up before the air cylinder pinches above the drop. The scale bar is $2 \mathrm{~mm}$. (b) Close-up images following the antibubble. The arrows highlight two prominent features in the interference patterns observed in the light transmitted through the antibubble. These patterns move up, indicating how the bottom section of the bubble shell thins. The scale bar is $1 \mathrm{~mm}$. (c) The contraction of the air layer at the top apex entraps a microdroplet of pool liquid inside the bubble, which is seen bouncing around inside the bubble (see the supplementary video). The scale bar is $1 \mathrm{~mm}$. 
(a)

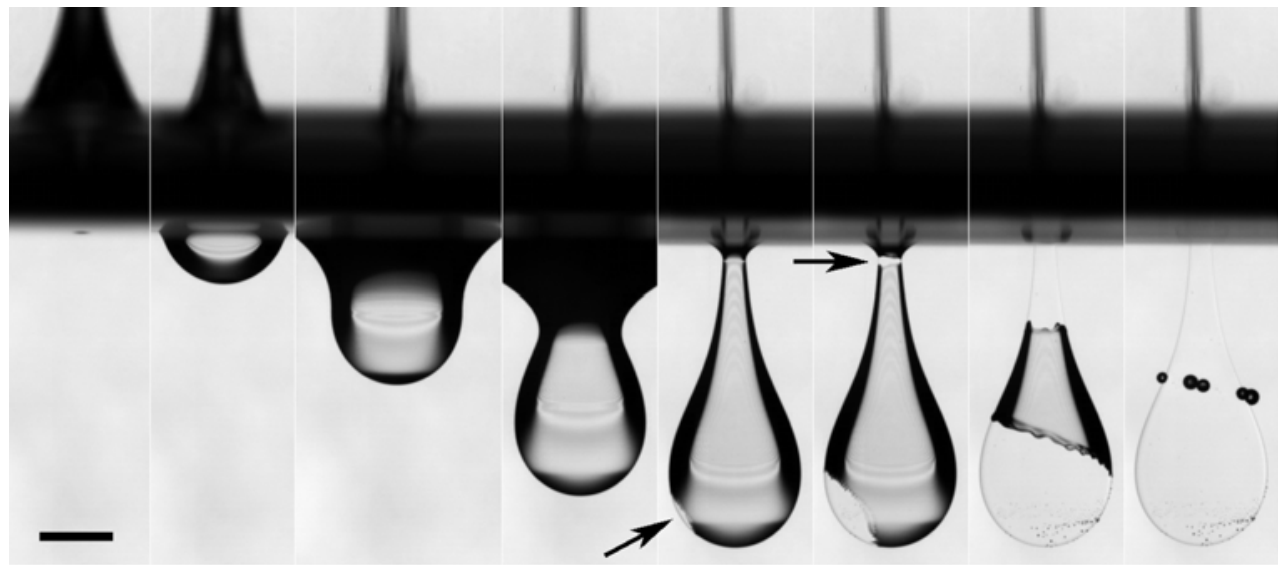

(b)
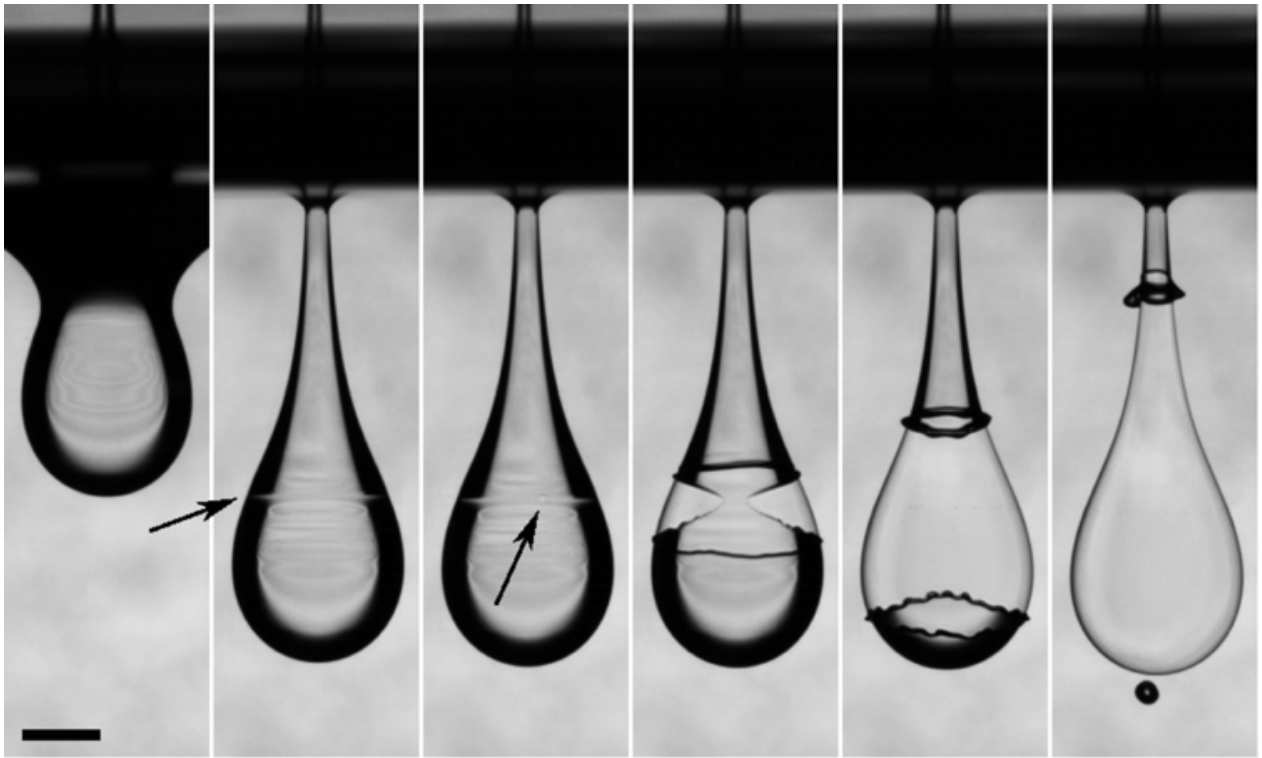

FIGURE 10. Examples of different rupture mechanisms of the air film wrapped around the drop and the trailing liquid thread. (a) Typical impact of a viscous drop when a liquid thread is attached to the nozzle. Here, the drop viscosity $v_{d}$ is $1000 \mathrm{cSt}$ and the pool viscosity $v_{p}$ is $5 \mathrm{cSt}, H=27.5 \mathrm{~mm}$. The images are $0,2,7,17,51.952 .3,53.4$ and $56 \mathrm{~ms}$ after first contact with the pool. The arrows point out the two rupture points. $(b)$ The air sheet breaks in a zipper-like fashion at an axisymmetric line pointed out by the first arrow. The rupture starts at the second arrow and runs around the periphery at an average velocity of $5.6 \mathrm{~m} \mathrm{~s}^{-1}$. Here, $v_{d}=10000 \mathrm{cSt}, v_{p}=0.65 \mathrm{cSt}, H=21.5 \mathrm{~mm}$. The images are 18, 65, 66.5, 66.9, 67.9 and $70.4 \mathrm{~ms}$ after first contact with the pool. The scale bars are both $1 \mathrm{~mm}$.

which is extracted from the video in figure $12(a)$. We also include an estimate of the film thickness based on the Taylor-Culick velocity, $\delta=2 \sigma /\left(\rho U^{2}\right)$. This thickness is not valid in the midsection, where the toroidal bubble slows the motion, but at the bottom and around the top thread, $\delta<500 \mathrm{~nm}$. It should be kept in mind that 
(a)

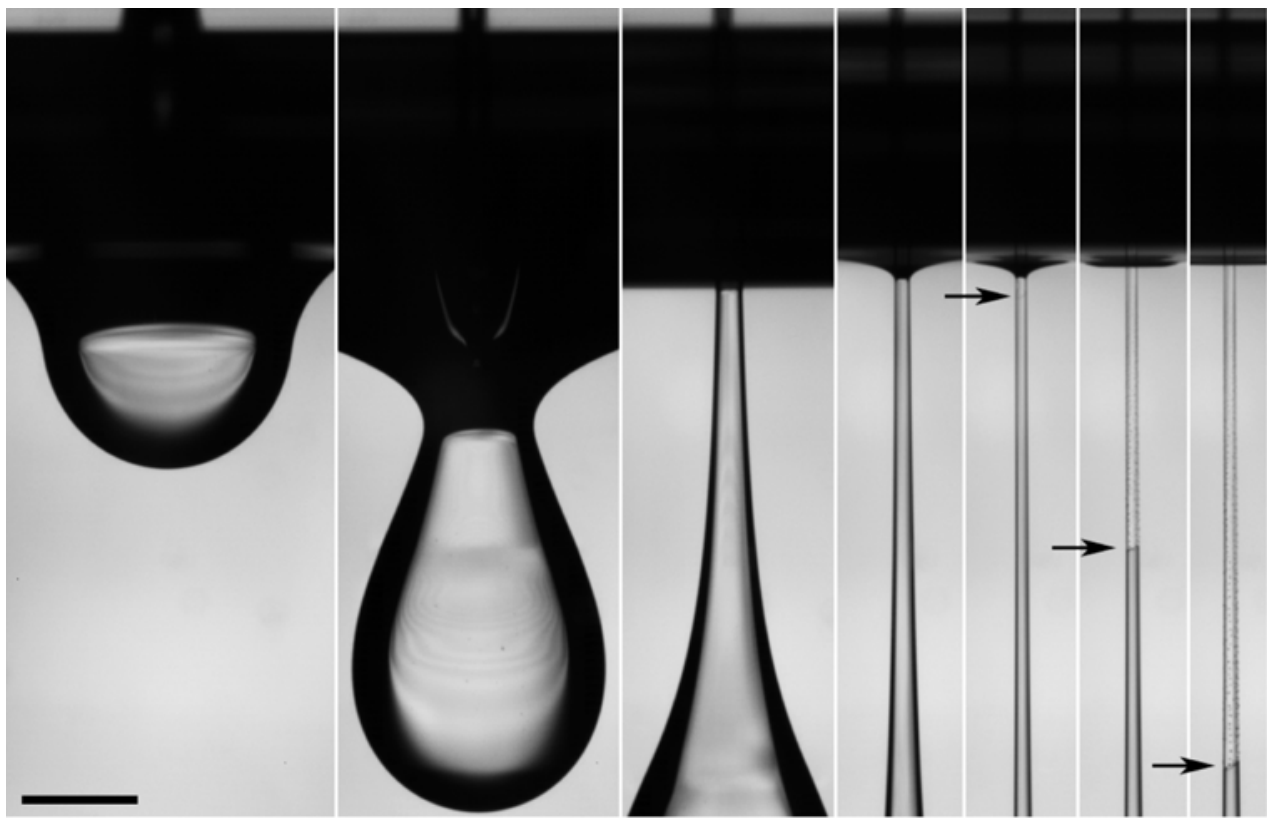

(b)

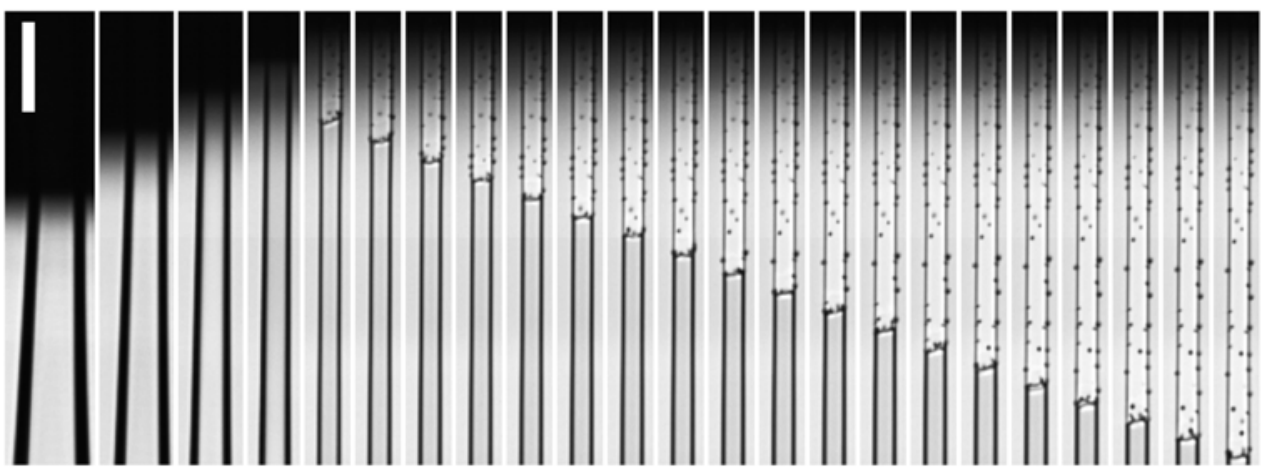

FIGURE 11. Example of cylinder rupture starting near the free surface. (a) Here, $v_{d}=$ $10000 \mathrm{cSt}$ and $v_{p}=0.65 \mathrm{cSt}, H=23 \mathrm{~mm}$. The images are 5.7, 21, 46, 86, 123.7, 124.1 and $124.5 \mathrm{~ms}$ after first contact with the pool. The arrows point out the rupture near the free surface and the air breakup front propagating down the thread, starting at $7 \mathrm{~m} \mathrm{~s}^{-1}$. The scale bar is $1 \mathrm{~mm}$. (b) Typical propagation of the rupture front, for $v_{d}=1000 \mathrm{cSt}$ and $v_{p}=1 \mathrm{cSt}, H=25 \mathrm{~mm}$. The first four images are $1.14,0.89,0.64$ and $0.39 \mathrm{~ms}$ before the rupture starts. The frames during the rupture are spaced by $10 \mu \mathrm{s}$. The scale bar is $200 \mu \mathrm{m}$.

for sufficiently thin air layers the pool viscosity will begin to become important, irrespective of how low $\mu_{p}$ is. The maximum edge velocity for a particular liquid is characterized by the capillary-viscous velocity $u_{\sigma \mu}=\sigma / \mu$. This is thereby an upper bound for the capillary-inertial Taylor-Culick velocity. Equating the two gives us an estimate of how thin the air layer can become before viscous forces dominate, giving $\delta_{\star}=2 \mu_{p}^{2} /(\rho \sigma)$. This critical thickness is listed in table 1 for the different liquids. For the pool viscosity in figure $12(c), v_{p}=1 \mathrm{cSt}$, we obtain $\delta_{\star}=94 \mathrm{~nm}$. One can therefore expect the rupture velocity to be slowed down by viscous effects for 
(a)

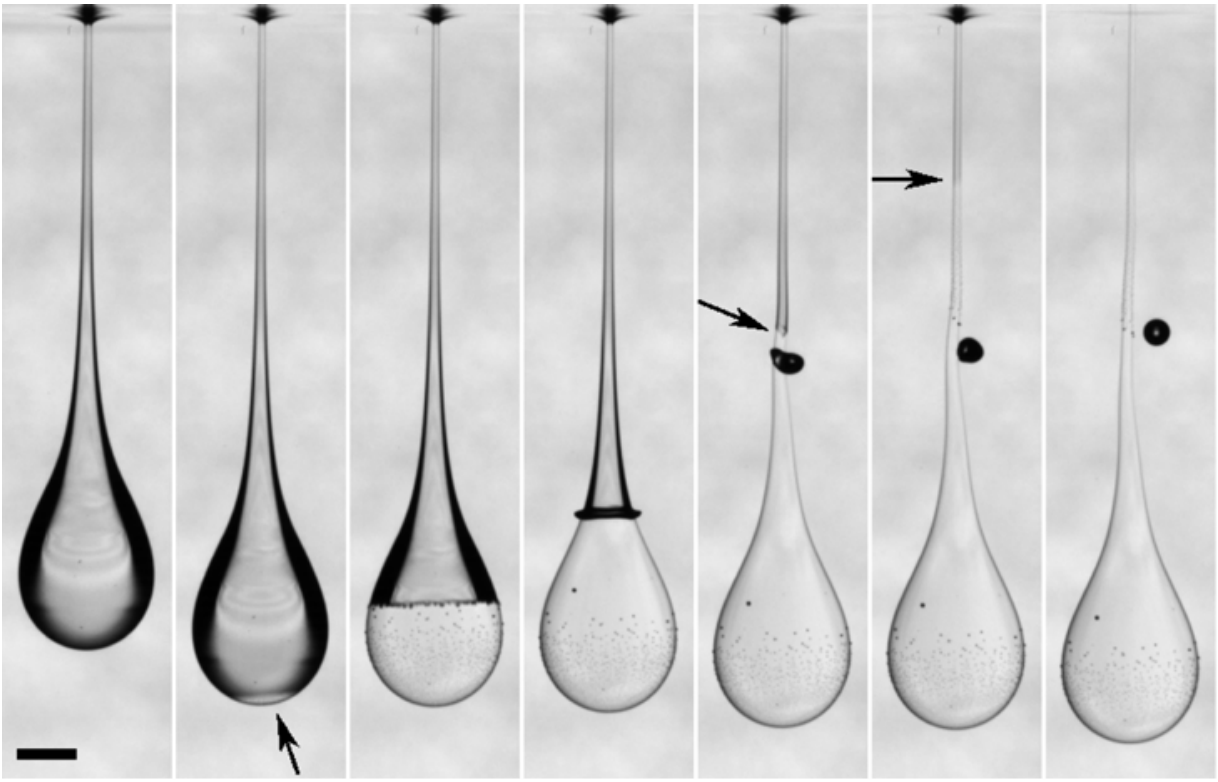

$\delta(\mu \mathrm{m})$
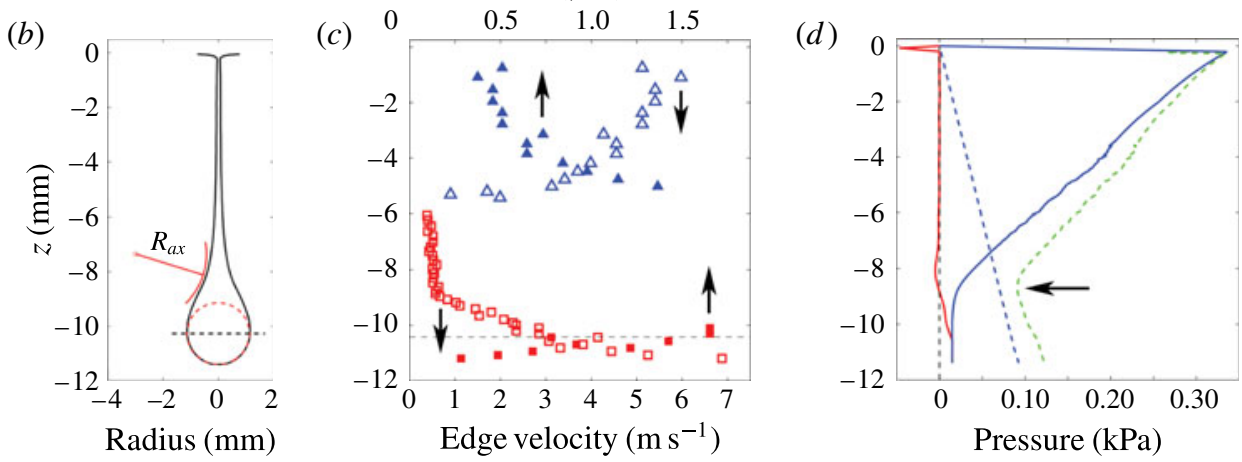

FIgURE 12. (Colour online) (a) Typical air layer enwrapping an impacting viscous drop along with a liquid thread that is attached to the nozzle. Here, the drop viscosity is $v_{d}=$ $10000 \mathrm{cSt}$ and the pool viscosity is $v_{p}=1 \mathrm{cSt}$, for an impact height of $H=24 \mathrm{~mm}$. The images are $-22,0.04,0.59,2.6,7.7,8.4$ and $12.9 \mathrm{~ms}$ after first rupture of the air film. The rupture occurs $144 \mathrm{~ms}$ after the drop first impacts the free surface. The arrows point out the bottom rupture point and the edge of film ruptures moving up along the thread. The scale bar is $1 \mathrm{~mm}$. (b) The shape of the air film, from $(a)$. (c) The edge rupture velocity versus the depth $z$. The blue triangles are for the rupture above the bubble, which starts when the bubble torus unwraps from the viscous thread. The dashed line shows the depth of the drop equator. The filled symbols show the air-film thickness $\delta$ estimated from the Taylor-Culick velocity, with values listed on the top axis. For the thinnest sections of the air film we expect this estimate to only be an upper bound for the thickness, as viscosity becomes important and slows down the motion of the edge. See also the direct measurements in figure 15. (d) Capillary and hydrostatic pressures: azimuthal (blue line) and axial (red line) capillary pressures, hydrostatic pressure (blue dashed line) and sum of all three (green dashed curve). 
(a)

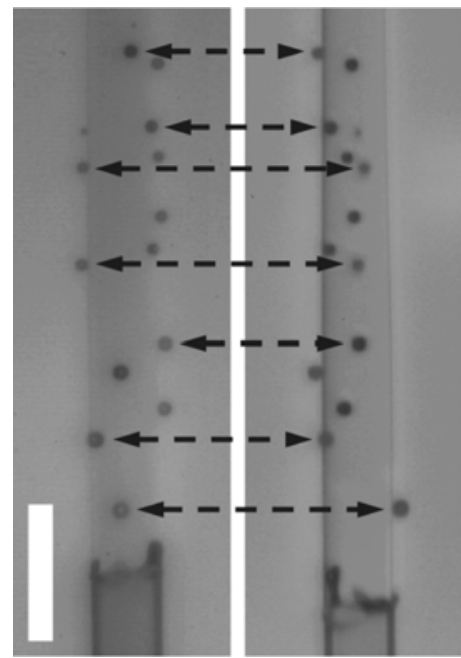

(b)

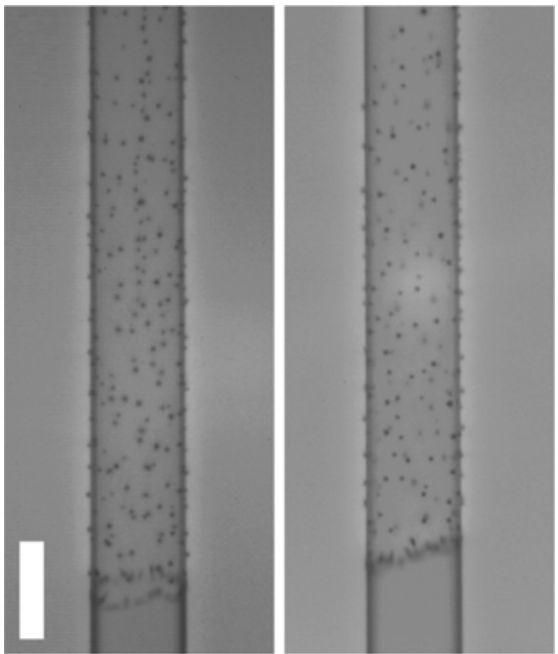

(c)

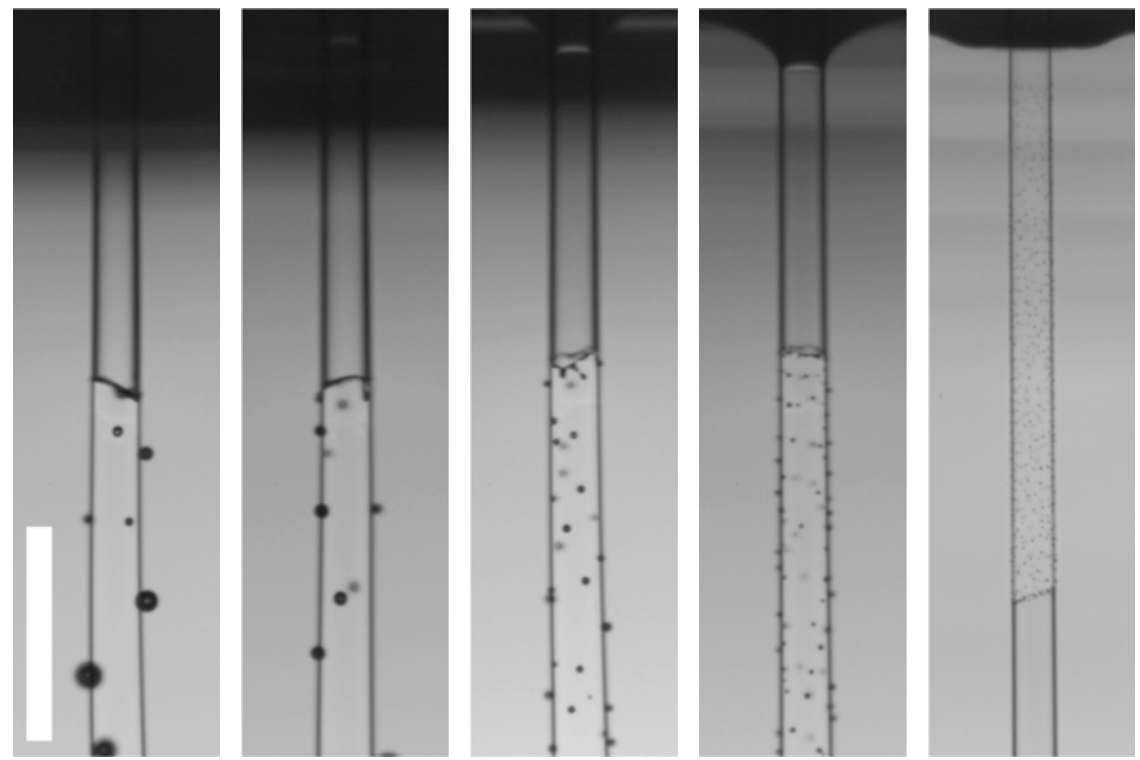

FIGURE 13. Microbubbles left behind following the ruptures of the air cylinders. (a) Two perpendicular camera views for $v_{d}=1000 \mathrm{cSt}, v_{p}=1 \mathrm{cSt}$ and $H=22 \mathrm{~mm}$. The thread diameter is $51 \mu \mathrm{m}$ and the breakup velocity is $u_{\text {edge }}=3.6 \mathrm{~m} \mathrm{~s}^{-1}$. (b) Two perpendicular camera views for $v_{d}=10000 \mathrm{cSt}, v_{p}=1 \mathrm{cSt}$ and $H=25.5 \mathrm{~mm}$. The thread diameter is $95 \mu \mathrm{m}$ and the breakup velocity is $u_{\text {edge }}=5.5 \mathrm{~m} \mathrm{~s}^{-1}$. (c) Microbubble sizes for the same liquids as in $(b)$, but for different impact conditions. From left to right $H=35,32$, 29, 27 and $25 \mathrm{~mm}$ and the corresponding breakup velocities are $u_{\text {edge }}=2.3,2.6,3.0,3.6$ and $5.8 \mathrm{~m} \mathrm{~s}^{-1}$. Based on the volume of the microbubbles we estimate the corresponding thicknesses of the air films to be $649,289,183,166$ and $75 \mathrm{~nm}$. The scale bars in $(a)$ and $(b)$ are $100 \mu \mathrm{m}$ and in (c) $500 \mu \mathrm{m}$.

the thinnest regions of the air layer, thereby overestimating somewhat those air-layer thicknesses in figure 12(c). For even thinner air layers this becomes much more pronounced, as will be shown in figure 15 . 


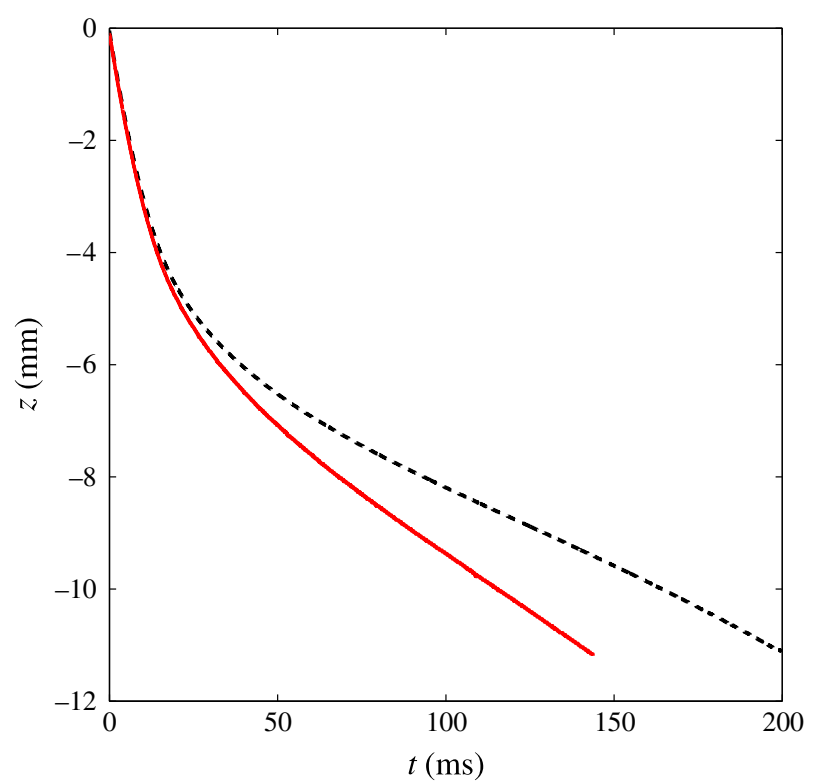

Figure 14. (Colour online) Penetration depth versus time for the impact conditions in figures 12 and 18.

Figure $12(d)$ shows the components of the capillary pressure, calculated from the two principal radii of curvature

$$
p_{\sigma}=\sigma\left(\kappa+\frac{\cos \theta}{R_{t}}\right),
$$

where $\kappa=1 / R_{a x}$ is the axial curvature, $R_{t}$ is the local radius of the air sheet and $\theta$ is the angle of the free surface to the vertical. The axial curvature is not well resolved at the pool surface, but is always much smaller than the azimuthal one, except near the spherical bottom surface where they are equal. It should be noted that the hydrostatic pressure $p_{h}=\rho g z$ inside the liquid cannot push out the air from the thin section of the thread as it is three times smaller than the capillary pressure. In fact there is a minimum in the sum $p_{\sigma}+p_{h}$ near where the thread meets the drop (arrow in figure $12 d$ ). The bottom section is close to a perfect hemisphere and thins by the weight of the inner drop, causing the bottom rupture. Figures $11(b)$ and 13 show close-up sequences of the breakup of the air cylinder for other impact conditions.

The finest microbubbles arise when the air cylinder breaks near the pool surface and the rupture travels downwards, whereas larger bubbles are produced when the air layer ruptures further down and the rupture travels up towards the pool surface, indicating that it has not thinned sufficiently to break on its own. Therefore, the air cylinder that ruptures downwards in figure $13(c)$ has the thinnest air film and smallest microbubbles.

The two-camera views described in figure 2(a) were used to measure accurately the volume of the microbubbles to find the air-film thicknesses, which are shown in figure 13(c). The thinnest film in that figure is estimated to be only $75 \pm 25 \mathrm{~nm}$ thick. Figure 15 uses these air-film thicknesses to compare the edge breakup velocities with the Taylor-Culick velocity $u_{\text {edge }}=C \sqrt{2 \sigma /(\rho \delta)}$. The results are best fitted with 


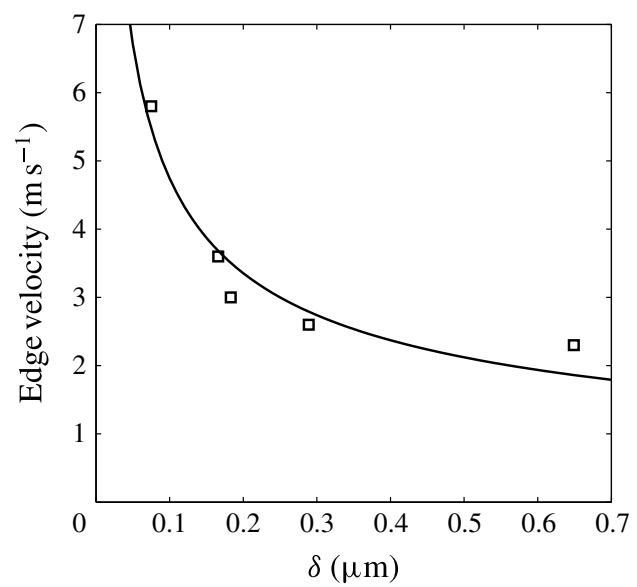

FIGURE 15. The edge breakup velocity of the very thin air films shown in figure 13(c) compared with the Taylor-Culick velocity $u_{e d g e}=C \sqrt{2 \sigma /(\rho \delta)}$, with the empirical constant $C=0.23$. The data are for $v_{d}=10000 \mathrm{cSt}$ and $v_{p}=1 \mathrm{cSt}$, over a range of different impact heights.

the empirical proportionality constant $C=0.23$, showing that the edge velocities are significantly slower than this inviscid prediction. This could be expected as the capillary-viscous velocity $u_{\sigma \mu}=\sigma / \mu$ of the $1 \mathrm{cSt}$ silicone oil is only $21 \mathrm{~m} \mathrm{~s}^{-1}$ and the smallest values of $\delta$ are of similar order to $\delta_{\star}=94 \mathrm{~nm}$. We must conclude that the functional form used in figure 15 is purely empirical, not arising from capillary-inertial dynamics. The rapid increase in the velocity for the thinnest film, $\delta \simeq 75 \mathrm{~nm}$, may have more to do with the nature of the continued rupturing of the air film; see the discussion in Thoroddsen et al. (2012) for a similar situation arising for highly viscous liquids. The very low Reynolds number in the extremely thin air layer may also help to slow down the motion and lead to its breakup.

\subsection{Interferometric measurements of the air-layer thickness}

In many of the video images we see interference patterns, which appear even for the broadband spectra of the metal-halide light. To better assess the thickness of the air film, we performed a separate set of experiments using a monochromatic pulsed light source, to allow for interferometry, as described in $\$ 2.4$. Such interference images are shown in figure 16(a). The azimuthal curvature of the thread distorts the fringe patterns and we only consider them along the symmetry axis. We use a frame rate of $30 \mathrm{kfps}$, which allows us to follow the individual fringes and keep track of the local thickness variations. The absolute thickness can be determined by the lack of fringes along the top section of the cylindrical thread, leading up to its rupture, see the second to last panel in the figure. We thereby conclude that the air-layer thickness along the top section of the air cylinder has become $\delta<160 \mathrm{~nm}$, which is consistent with the estimate based on the microbubble volumes in the previous section.

In figure $16(b)$ we show the thickness variation measured from these images. It should be kept in mind that the total vertical extent of the view is here limited, due to the high frame rates and the need for sufficient magnification to accurately resolve the fringes. In other sequences we observe the entire drop and air cylinder, but lose the resolution in the region with the largest number of fringes. This is 
(a)

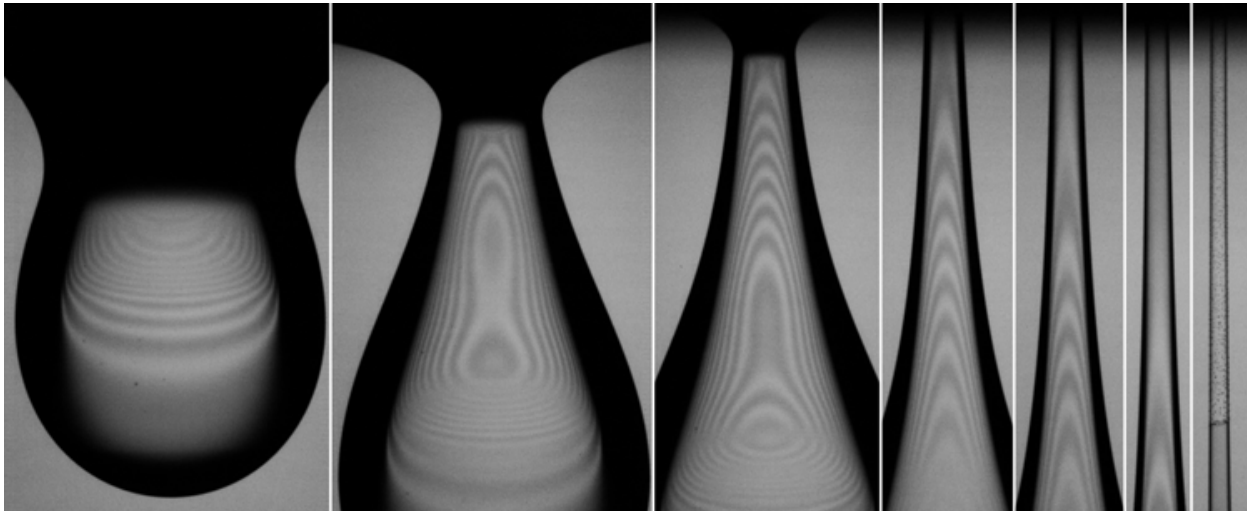

(b)

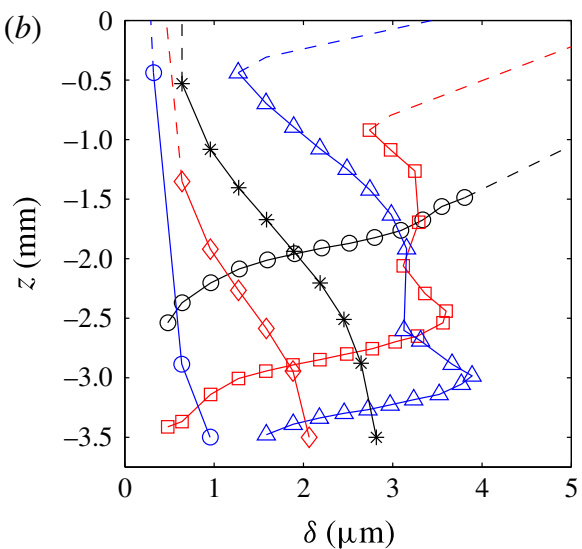

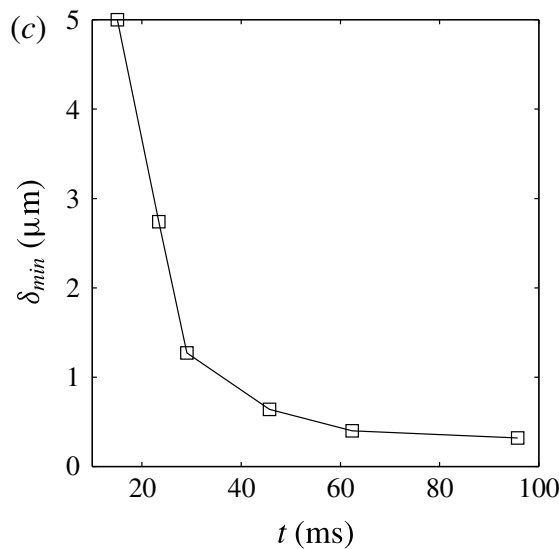

FIGURE 16. (Colour online) Interferometric fringes showing variation in air-film thickness along the drop, for $v_{d}=10000 \mathrm{cSt}, v_{p}=1 \mathrm{cSt}$ and $H=25.5 \mathrm{~mm}$. The illumination is monochromatic with $\lambda=640 \mathrm{~nm}$. The film thickness therefore changes by $\lambda / 4=160 \mathrm{~nm}$ between the centres of a bright and a dark fringe. The relative times shown are $t=0,8.3$, $14,30.7,47.3,80.7$ and $150.7 \mathrm{~ms}$. The last frame shows the rupture travelling down the thread, leaving microbubbles. $(b)$ The evolution of the air-layer thickness from the images in $(a)$. (c) The time evolution of the air thickness in the throat where the air cylinder enters the pool.

shown in figure 17, which includes a separate close-up video frame taken at higher magnification. In this way we can combine imaging at two different magnifications, to resolve the entire thickness profile, assuming repeatability from one realization to the next (see the supplementary material). It should be kept in mind that the large surface slope at the bottom obscures the fringes, not allowing measurement of the $\delta$ there. In many cases there is a thicker section near the bottom, see Thoroddsen et al. (2012). The resulting time evolution of the air-layer thickness profiles for the case in figure $18(a)$, where the thread ruptures near the pool, is shown in figure $18(c)$. The local maximum in $\delta$ descends into the pool and becomes more pronounced, whereas the air layer near the pool thins rapidly, as was seen in figure $16(c)$. The growth in the maximum thickness is partly due to the decrease in the local radius of the droplets, but may also indicate a flow within the air film towards the minimum in the overall pressure, which was shown in figure $12(d)$. The bubbles left over at the 


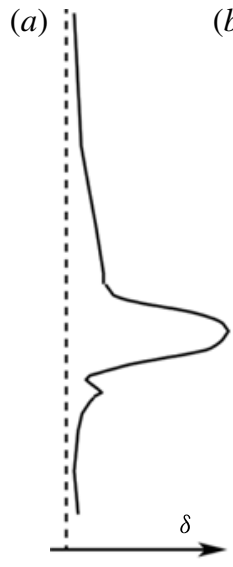

(b)
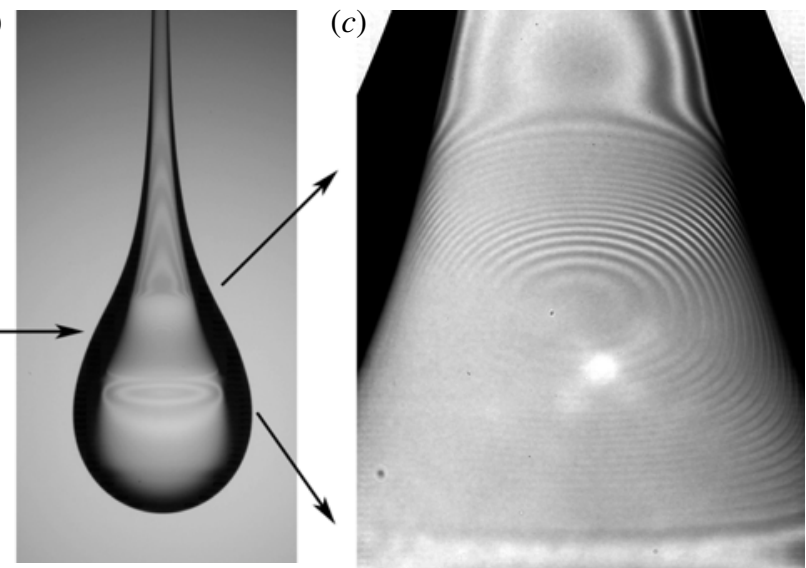

(d)
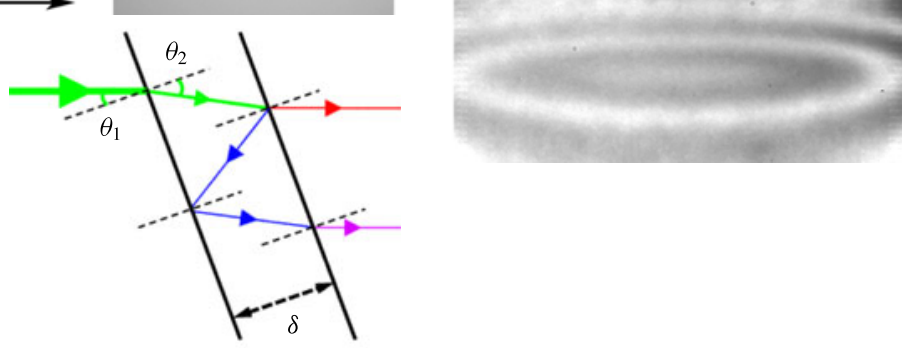

FIGURE 17. (Colour online) Interferometric fringes showing variation in the air-film thickness along the drop, for $v_{d}=10000 \mathrm{cSt}, v_{p}=1 \mathrm{cSt}$ and $H=25.5 \mathrm{~mm}$. Panel $(c)$ is from a higher-magnification realization, and shows a close-up of the blurred region in $(b)$. The blurriness arises from insufficient pixel resolution. Sketch $(a)$ shows the air-layer thickness $\delta$ profile, measured from the combined fringes from both images. The maximum air-layer thickness is here $\delta \simeq 6.5 \mu \mathrm{m}$. $(d)$ Explains how diffraction affects the interference measurements.

bottom of the drop in the last panel of figure 18(a), following the rupture, can be used to support the interference measurements. We do this by integrating the $\delta$-profile to obtain the air volume. We find correspondence within $20 \%$, which is acceptable, as we cannot accurately estimate the air thickness under the bottom of the drop.

\section{Changing the drop size}

The parameter spaces presented in figures 4 and 5 were mapped out using the same nozzle, thereby producing similar drop sizes in all cases, and the $O h_{d}$ only varied due to different viscosity of the drop liquid. However, we do not expect the parameter space to collapse only with $O h_{d}$, as the drop size and structure of the viscous thread connecting the drop to the nozzle will certainly depend on the Bond number, $B o=\rho_{d} g R^{2} / \sigma$, of the pinch-off process. In other words, the $W e_{p}-O h_{d}$ regime diagrams cannot be universal. We therefore explore, in this section, the changes in the phenomena as we use a range of different droplet sizes, over a limited range of different viscosities. The overall behaviour was similar to before, but the details varied greatly. Figure 19 shows three different drop sizes, while keeping the drop viscosity fixed. We used nozzles with diameters from $0.4 \mathrm{~mm}$ to $5 \mathrm{~mm}$, giving drop diameters $D$ from 1.65 to $2.83 \mathrm{~mm}$, which are the limiting drop sizes possible in our set-up. 
(a)
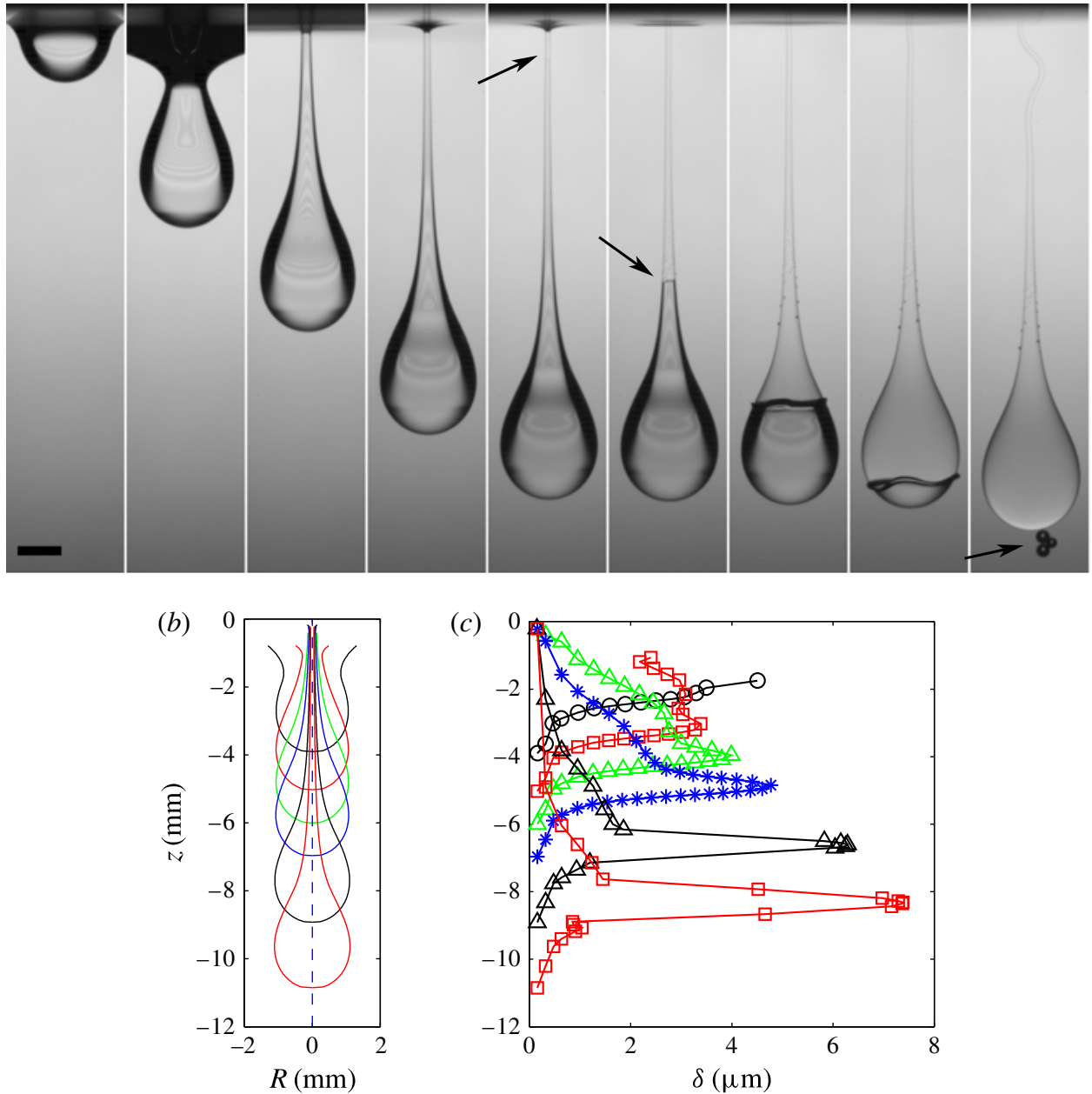

FIgURE 18. (Colour online) The evolution of the air layer, for $v_{d}=10000 \mathrm{cSt}, v_{p}=$ $1 \mathrm{cSt}$ and $H=25.5 \mathrm{~mm}$. (a) Overall evolution, shown at $t=4,21,64,144,192.65,193.9$, 196.15, 198.9 and $212.3 \mathrm{~ms}$. The first arrow points to the rupture point near the pool surface. The scale bar is $1 \mathrm{~mm}$. See also the supplementary movies. (b) Drop shapes at $t=15,25,40,62.5,127.5$ and $192.6 \mathrm{~ms}$; the corresponding air-layer thickness profiles are shown in $(c)$.

This has little effect on $O h_{d}$ in figure 4, as its value is primarily determined by the drop viscosity. However, by simply changing $D$ we see both antibubbles and fine threads with cylindrical air layers, for very similar $O h_{d} \simeq 24$ and 19 respectively.

Changing the drop viscosity for the largest drops, $D=2.83 \mathrm{~mm}$, reveals qualitatively different thread shapes. In figure $20(a)$ we show a case where the fine viscous thread starts to pinch near the drop, retaining the thin air layer. The air layer ruptures while the very thin viscous thread remains intact, as is clear in the close-up sequence near the rupture point in figure $20(c)$. Here, the narrowest diameter at rupture becomes $39 \mu \mathrm{m}$. The edge of the air film, below the rupture point, moves downwards at $10.6 \mathrm{~m} \mathrm{~s}^{-1}$, which is the largest velocity measured herein. 


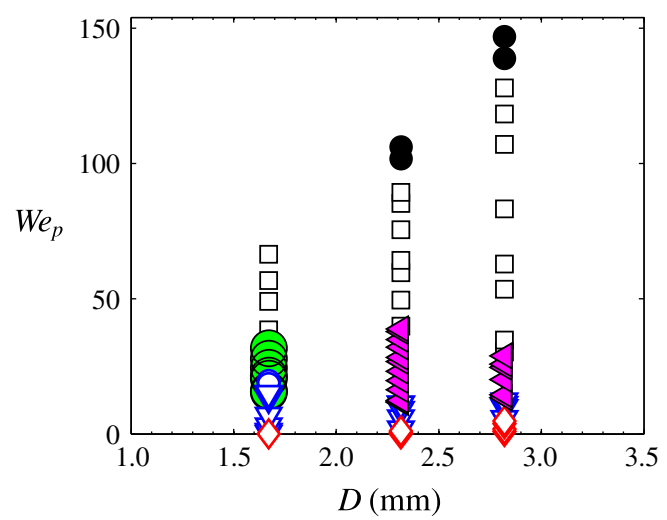

FIGURE 19. (Colour online) The parameter space for three different nozzle/drop sizes, for $v_{d}=3000 \mathrm{cSt}, v_{p}=1 \mathrm{cSt}$. The symbols have the same meaning as in figure 4.

Figure 21 shows a near spherical drop with a straight cylinder above it. The zipper rupture occurs along a sharp line on the drop. The close-up images in the two centre rows show how the ring of bubbles is formed. The interference patterns indicate a slightly thicker air layer below the zipper line. This air volume collects into a thick rim, which encounters a much thinner air layer below it. The rupture moves much faster in this thinner layer and breaks through the bubble, leaving a myriad of very small bubbles below it. The thick cylinder is left behind, but is unstable by RayleighPlateau instability and breaks into regular uniform-sized bubbles. Near the bottom of the drop there is a much darker section, which we believe is thicker (see Thoroddsen et al. 2012). When the fast moving rupture front meets the edge of this thicker layer, it slows down and breaks up into slightly larger bubbles. Figure 21(b) shows a slightly different impact height, where the initial zipper leaves a line of fine microbubbles, in addition to the equator and bottom bubbles.

The thin zipper line arises from the initial wrapping of the free surface about the upper section of the drop.

\section{Discussion and conclusions}

\subsection{Stability of the cylindrical air films}

In contrast to the antibubbles, which are minimal surfaces, the cylindrical air sheet remains stable over an extended period of time, longer than one might expect, due to the intrinsic instability and rapid breakup of fine hollow tubes of air and low-viscosity liquid jets (Chandrasekhar 1961). In fact, we never observe a typical periodic capillary breakup of the cylindrical air layer, as would be expected from inviscid Rayleigh-type instability, as for example is quite evident for hollow air cylinders during the pinchoff of a bubble (Burton, Waldrep \& Taborek 2005; Thoroddsen, Etoh \& Takehara 2007). Here, the difference is of course the presence of the inner viscous core, which restricts the motion within the air layer through viscous forces. The linear stability of gas cylinders ignores the dynamics of the inner gas, assigning all the inertia to the much denser outer liquid (Chandrasekhar 1961, p. 540). To better characterize the stability of our cylindrical air layers, we measure the time interval from the start of cylinder formation, i.e. when cylinder length exceeds the periphery of the cylinder; in 
(a)

(b)
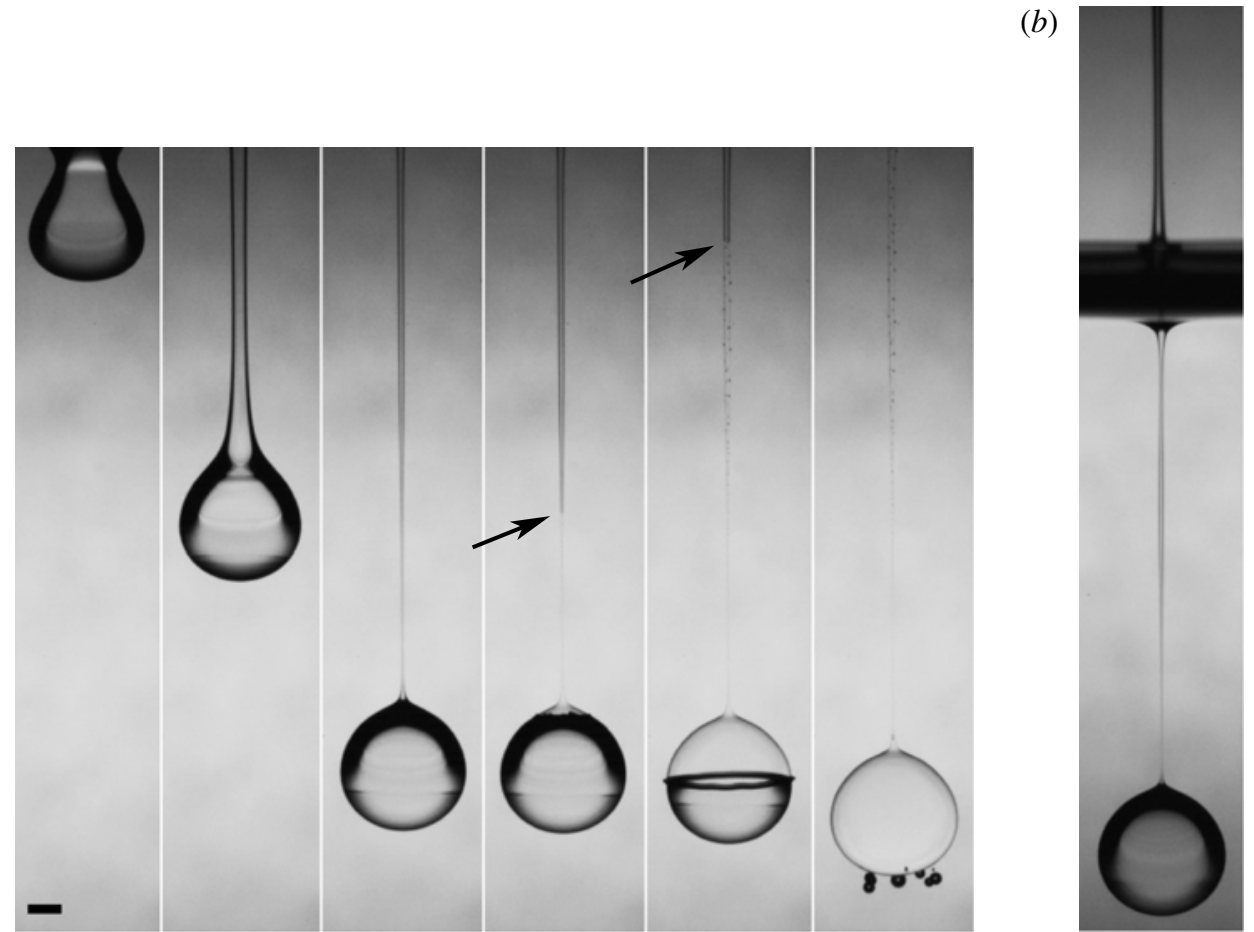

(c)
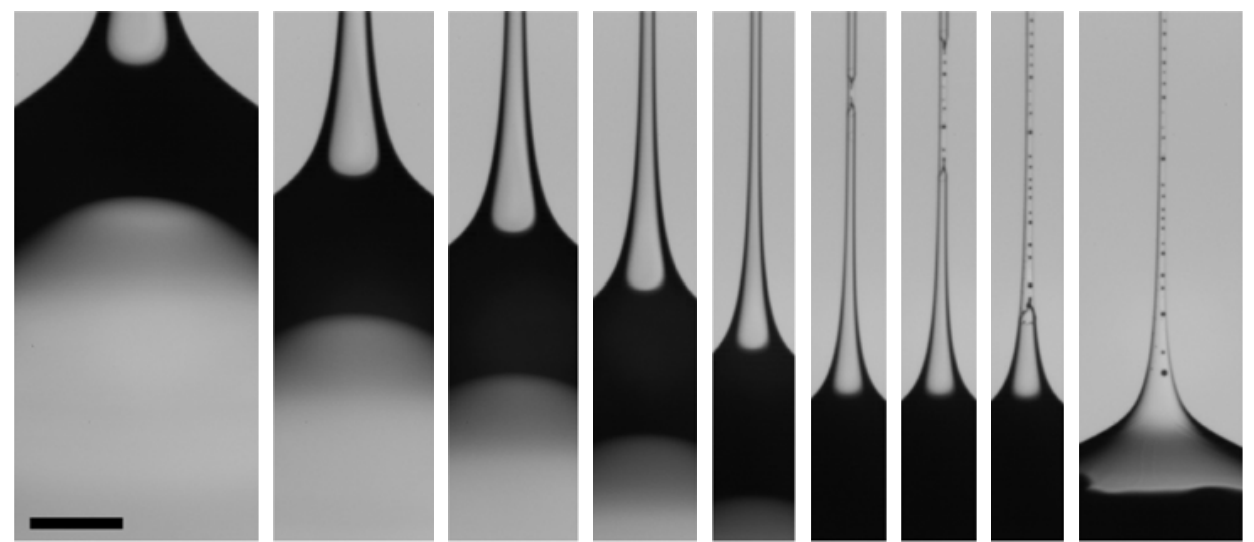

FIGURE 20. Formation of a very long and slender air cylinder for a large drop, $D \simeq$ $2.83 \mathrm{~mm}$, for $v_{d}=500 \mathrm{cSt}, v_{p}=1 \mathrm{cSt}$ and $H=23 \mathrm{~mm}$. (a) The times shown are $t=$ $30.85,97.50,178.68,179.55,182.88$ and $194.30 \mathrm{~ms}$ after the drop reaches the pool surface. The slender air cylinder is intact in the third panel and breaks near its thinnest section before the fourth panel. The scale bar is $1 \mathrm{~mm}$. (b) Image from a separate realization, showing the intact air layer as well as the liquid thread above the pool surface, taken at $t=198 \mathrm{~ms}$ after the drop starts deforming the pool. The viscous thread thickens above the pool, due to capillary resistance, while penetrating the free surface. (c) Stretching and breakup of the long and slender air cylinder, under the same conditions as above. The times relative to the rupture are $t=-19.1,-12.5,-9.1,-5.8,-2.5,0,0.033,0.167$ and $6.4 \mathrm{~ms}$. The scale bar is $500 \mu \mathrm{m}$. 
(a)
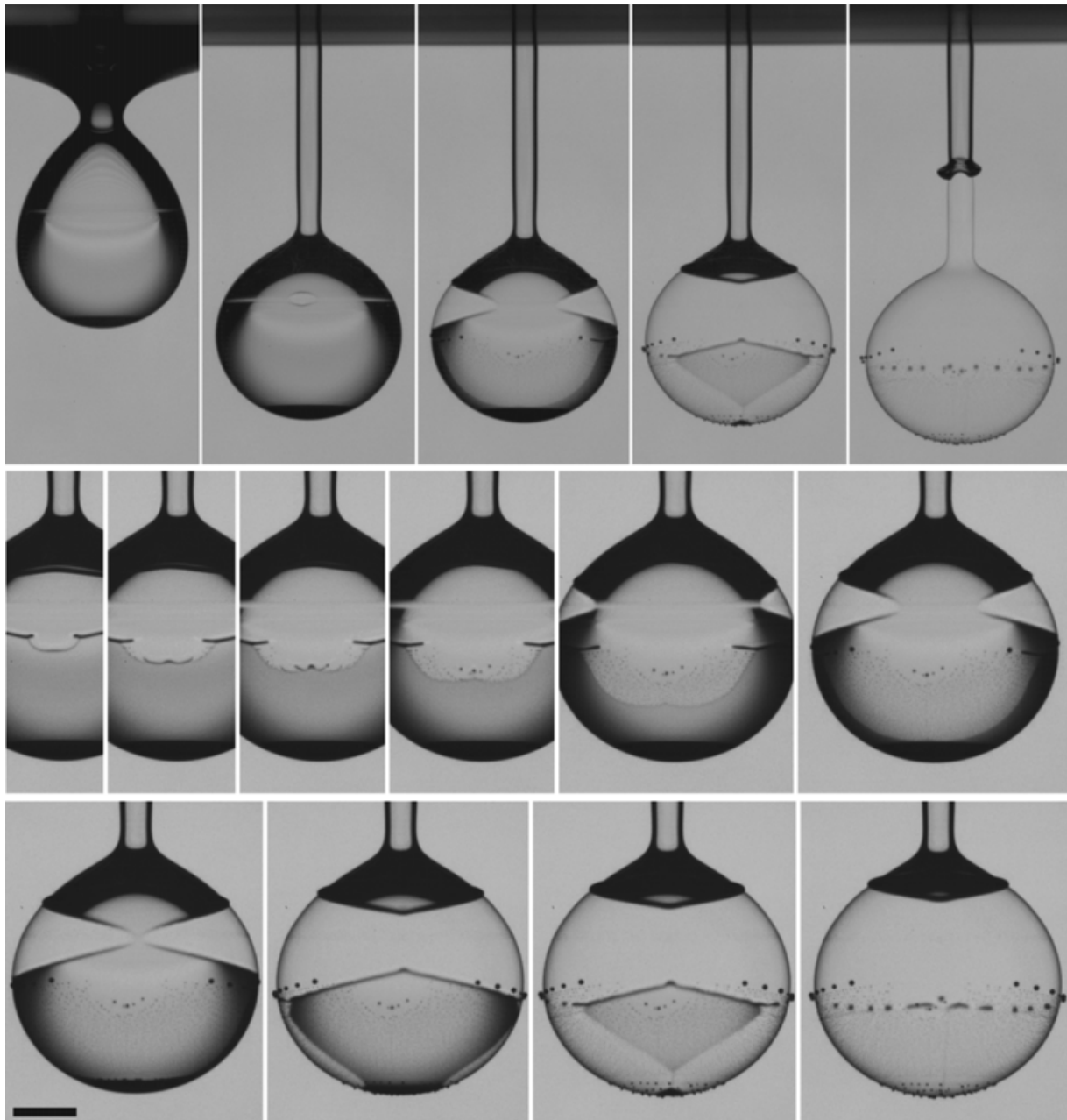

(b)

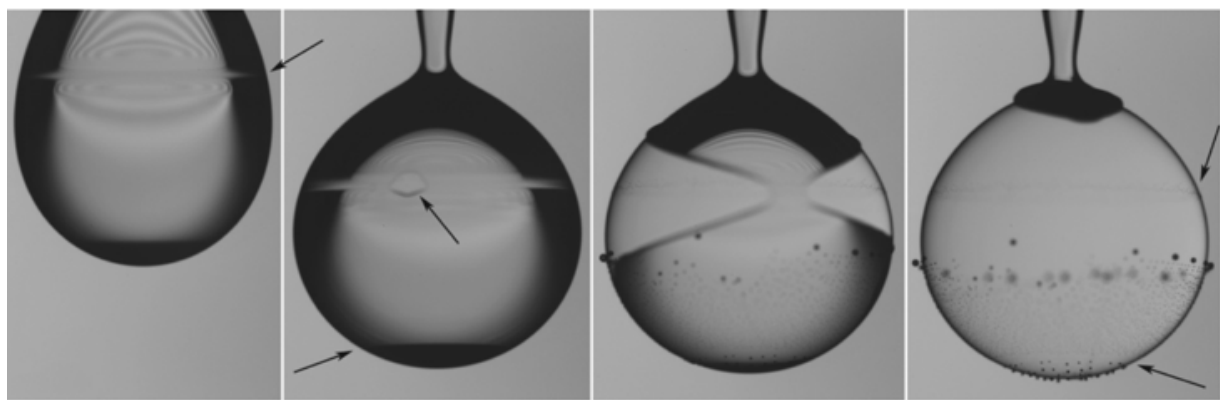

FIGURE 21. Formation of an azimuthal ring of microbubbles around the equator of the drop, $D \simeq 2.8 \mathrm{~mm}$, for $v_{d}=100 \mathrm{cSt}, v_{p}=1 \mathrm{cSt}$ and $H=18 \mathrm{~mm}(a)$. Top row: $t=34,60$, 60.7, 61.4 and $66.4 \mathrm{~ms}$. Centre two rows: close-ups at $t=0.40,0.50,0.55,0.60,0.70$, $0.85,0.95,1.25,1.40$ and $1.60 \mathrm{~ms}$ after rupture. The scale bar is $1 \mathrm{~mm}$. (b) Slightly different release height of $H=16.5 \mathrm{~mm}$, which also shows clear microbubbles along the initial zipper. 
other words, when the aspect ratio is sufficiently large to satisfy the Rayleigh-Plateau instability criterion. The characteristic breakup time for linear capillary instability of a hollow air cylinder of radius $R_{\text {air }}$ is

$$
\tau_{\sigma}=\frac{1}{0.8201} \sqrt{\frac{\rho R_{\text {air }}^{3}}{\sigma}} .
$$

The longest and most regular air cylinders around the viscous threads are observed for the highest drop viscosity of $v_{d}=10000 \mathrm{cSt}$, as shown in figure 12, where $R_{\text {air }}=50 \mu \mathrm{m}$, which within the pool of silicone oil gives $\tau_{\sigma}=95 \mu \mathrm{s}$. For the conditions in figure 12, the air cylinder remains stable for at least $121 \mathrm{~ms}$, before it breaks up due to the travelling ruptures and not to a varicose capillary instability along its length. This duration is at least three orders of magnitude longer than $\tau_{\sigma}$. For a lower-viscosity drop of $v_{d}=1000 \mathrm{cSt}$ (figure $11 \mathrm{~b}$ ), the thread can become even thinner, $R_{\text {air }}=25 \mu \mathrm{m}$, giving an even shorter $\tau_{\sigma}=34 \mu \mathrm{s}$. Therefore, other effects must stabilize this breakup. Below, we discuss some possibilities in turn. Recent studies on linear stability of stationary compound cylinders are due to Chauhan et al. (2000) and Liang et al. (2011). Our system is isothermal, ruling out thermocapillary effects (Neitzel \& Dell'Aversana 2002).

Axial stretching can stabilize jets (Tomotika 1936). However, our fine cylindrical sheets remain stable, until the end, when the downwards motion has almost ceased. This is the case in figure 12, where the drop has essentially stopped moving when the breakup travels up the thread, indicating that stretching is not the only factor stabilizing the air cylinder.

Axial air flow within the sheet can be set up by the pressure gradients $\mathrm{d} p / \mathrm{d} z$ along the thread, shown in figure $12(d)$. The strength of this flow, $u_{\text {air }}$, can be estimated by balancing $\mathrm{d} p / \mathrm{d} z$ with the viscous stress in the layer of thickness $\delta$. For $\delta \sim 500 \mathrm{~nm}$, this gives $u_{\text {air }}=-(\mathrm{d} p / \mathrm{d} z) \times(\delta / 2)^{2} /\left(2 \mu_{\text {air }}\right) \simeq 0.17 \mu \mathrm{m} \mathrm{ms}^{-1}$, which is weak on the time scales involved. The corresponding ratio of dynamic to capillary pressure in the thread is $p_{d y n} / p_{\sigma} \sim 10^{-10}$. We can conclude that the local thickness of the air layer is established early on by the original wrapping of the free surface around the drop and by the subsequent stretching of this surface. Only in the thickest sections of the air film, measured in figures 17 and 18, where $\delta \sim 5 \mu \mathrm{m}$, can we expect 100 times higher $u_{\text {air }}$, which may be sufficient to redistribute the air. This view of frozen localized air-layer thickness is supported by the zipper breakup in figure 21 , where the thin horizontal line is produced at the pool surface and persists as the drop penetrates below the free surface, where it eventually ruptures.

Axial shear across the air sheet may affect the air-flow dynamics. Here, pool liquid is pulled down with the thread, transmitting the stress across the air layer. However, immediately following its breakup the microbubbles can be tracked, as they are probably attached at the interface of the two liquids. These bubbles show only minor downwards motion, reducing the significance of this effect.

Lubrication in the air layer would certainly slow down any varicose motions, with the small $R e$ of flow within a submicron $\delta$ being viscous dominated. We showed that the thickness of the air layer reaches down to less than $100 \mathrm{~nm}$. However, the thickness of the air layer during the stretching of the viscous thread changes and is a constant fraction of the thread radius, i.e. $\delta / R_{t}(t)=$ const. It should be kept in mind that some air could be entrained along with the thread, like for a plunging jet (Ohl, Oguz \& Prosperetti 2000; Kersten, Ohl \& Prosperetti 2003; Lorenceau, Quéré \& Eggers 2004), but the jet decelerates rapidly before it ruptures and the neck connecting 
to the pool becomes very narrow. Lubrication, possibly in combination with the above effects, must stabilize the air during this entire process. For the stretching thread in figure $11(b), \delta$ thereby reduces from 650 to $200 \mathrm{~nm}$. Similarly, from the second to the fifth panel in figure $11(a), \delta$ goes from $1.9 \mu \mathrm{m}$ to $200 \mathrm{~nm}$. Determination of the original thickness of the layer, when the sheet wraps around the thread, would therefore determine whether viscous lubrication is dominant from the very start. The original thickness is set when the cavity encloses and embraces the viscous thread. The final embrace is set by surface tension and air-layer lubrication. This occurs when the neck travels upwards towards the free surface of the pool, like in figure 10. The interferometry results in figure 16(c) show that the air layer, near the pool surface, approaches $1 \mu \mathrm{m}$ thickness after the first $20 \mathrm{~ms}$. This will allow air exchange during the early stage of the film formation.

Conceptually, we expect the viscosity in the air layer to only slow down the breakup, but not fully stabilize it. One measure of this slowdown is a time scale based on the fastest-growing wavelength in (5.1), $\lambda^{*}=2 \pi R_{t} / 0.484$, and the capillary-driven lubrication air velocity, $u_{\sigma} \sim\left(\Delta p / 0.5 \lambda^{*}\right) \times(\delta / 2)^{2} /\left(2 \mu_{\text {air }}\right)$, in which $\Delta p \sim \sigma\left(\delta / R_{t}^{2}\right)$. This combines to a time scale $t_{\sigma}=0.5 \times \lambda^{*} / u_{\sigma}=337 \mu_{\text {air }} R_{t}^{4} /\left(\sigma \delta^{3}\right) \sim 100 \mathrm{~ms}$ (for $\delta=1 \mu \mathrm{m}$ and $R_{t}=25 \mu \mathrm{m}$ ), which is sufficient to stabilize the air layer over the experimental duration. However, additional surface forces could also help to counteract the capillary instability. Electrostatic forces could play a role, considering the low conductivity of silicone oils. Eventually, however, van der Waals forces should destabilize the very thin air film, see Li et al. (2014b).

\subsection{Future directions}

Future work should use colour interferometry to measure the air-film thickness directly with an even finer resolution than was possible herein, see De Ruiter et al. (2012). Colour interferometry is a powerful technique, where absolute thicknesses can be measured directly, with a stated accuracy of $30 \mathrm{~nm}$ for flat surfaces. It would be of interest to apply interferometry to thin air layers which have been observed to arise in many other dynamical systems. One recent example is the air film under a leaping shampoo jet (Lee et al. 2013), where the shear and surfactants stabilize the layer, which is approximately $500 \mathrm{~nm}$, estimated from bubble volumes.

Changing the gas composition or the atmospheric pressure would also be of interest in future experiments on these films.

\section{Acknowledgements}

The work described herein was supported by KAUST research funding. D.B. and A.G. were interns in KAUST's High-Speed Fluids Imaging Laboratory.

\section{Supplementary data}

Supplementary data is available at http://dx.doi.org/10.1017/jfm.2015.335.

\section{REFERENCES}

Brenner, M. P. \& Gueyffier, D. 1999 On the bursting of viscous films. Phys. Fluids 11 (3), 737-739.

Burton, J. C., Waldrep, R. \& TABorek, P. 2005 Scaling and instabilities in bubble pinch-off. Phys. Rev. Lett. 94, 184502. 
Chan, D. Y. C., Klaseboer, E. \& Manica, R. 2011 Film drainage and coalescence between deformable drops and bubbles. Soft Matt. 7, 2235-2264.

Chandrasekhar, S. 1961 Hydrodynamic and Hydromagnetic Stability. Dover.

Chauhan, A., Maldarelli, C., Papageorgiou, D. T. \& Rumschitzki, D. S. 2000 Temporal instability of compound threads and jets. J. Fluid Mech. 420, 1-25.

Couder, Y., Fort, E., Gautier, C.-H. \& Boudaoud, A. 2005 From bouncing to floating: noncoalescence of drops on a fluid bath. Phys. Rev. Lett. 94 (17), 177801.

Deng, D. S., Nave, J.-C., Liang, X., Johnson, S. G. \& Fink, Y. 2011 Exploration of in-fiber nanostructures from capillary instability. Opt. Express 19, 16273-16290.

De Ruiter, J., OH, J. M., VAn Den Ende, D. \& Mugele, F. 2012 Dynamics of collapse of air films in drop impact. Phys. Rev. Lett. 108, 074505.

Dorbolo, S., Reyssat, E., Vandewalle, N.\& QuÉré, D. 2005 Aging of an antibubble. Europhys. Lett. 69 (6), 966-970.

Dorbolo, S., Terwagne, D., Delhalle, R., Dujardin, J., Huet, N., Vandewalle, N. \& DenKov, N. 2010 Antibubble lifetime: influence of the bulk viscosity and of the surface modulus of the mixture. Colloids Surf. A 365, 43-45.

Etoh, T. G., Poggemann, D. \& Kreider, G. et al. 2003 An image sensor which captures 100 consecutive frames at 1000000 frames/s. IEEE Trans. Electron Devices 50, 144-151.

Kersten, B., Ohl, C. D. \& Prosperetti, A. 2003 Transient impact of a liquid column on a miscible liquid surface. Phys. Fluids 15, 821-824.

Kim, P. G. \& Stone, H. A. 2008 Dynamics of the formation of antibubbles. Europhys. Lett. 83, 54001.

Lee, S., Li, E. Q., Marston, J. O., Bonito, A. \& Thoroddsen, S. T. 2013 Leaping shampoo glides on a lubricating air layer. Phys. Rev. E 87, 061001.

Lhuissier, H. \& VillermauX, E. 2012 Bursting bubble aerosols. J. Fluid Mech. 696, 5-44.

Liang, X., Deng, D. S., NAVE, J.-C. \& Johnson, S. G. 2011 Linear stability analysis of capillary instabilities for concentric cylindrical shells. J. Fluid Mech. 683, 235-262.

Li, E. Q., Al-Otaibi, S. A., Vakarelski, I. U. \& Thoroddsen, S. T. 2014a Satellite formation during bubble transition through an interface between immiscible liquids. J. Fluid Mech. 744, R1.

Li, E. Q. \& Thoroddsen, S. T. 2013 The fastest drop climbing on a wet conical fiber. Phys. Fluids 25, 052105.

Li, E. Q., Vakarelski, I. U., Chan, D. Y. C. \& Thoroddsen, S. T. $2014 b$ Stabilization of thin liquid films by repulsive van der Waals force. Langmuir 30, 5162-5169.

Liow, J.-L. \& COLE, D. E. 2007 Bubble entrapment mechanisms during the impact of a water drop. In Proceedings of the 16th Australasian Fluid Mechanics Conference, Gold Coast, Australia, pp. 866-869.

Lorenceau, E. \& Quéré, D. 2004 Drops on a conical wire. J. Fluid Mech. 510, 29-45.

Lorenceau, E., QuÉrÉ, D. \& EgGers, J. 2004 Air entrainment by a viscous jet plunging into a bath. Phys. Rev. Lett. 93, 254501.

Mills, B. H., Saylor, J. R. \& Testik, F. Y. 2012 An experimental study of Mesler entrainment on a surfactant-covered interface: the effect of drop shape and Weber number. AIChE J. $\mathbf{5 8}$ (1), 46-58.

MolaceK, J. \& Bush, J. W. M. 2013 Drops bouncing on a vibrating bath. J. Fluid Mech. 727, $582-611$.

Neitzel, G. P. \& Dell'Aversana, P. 2002 Noncoalescence and nonwetting behavior of liquids. Annu. Rev. Fluid Mech. 34, 267-289.

Oguz, H. N. \& Prosperetti, A. 1989 Surface-tension effects in the contact of liquid surfaces. J. Fluid Mech. 203, 149-171.

Ohl, C. D., Oguz, H. N. \& Prosperetti, A. 2000 Mechanism of air entrainment by a disturbed liquid jet. Phys. Fluids 12, 1710-1714.

REYSSAT, É. \& QUÉRÉ, D. 2006 Bursting of a fluid film in a viscous environment. Europhys. Lett. 76 (2), 236-242.

SAYLOR, J. R. \& Bounds, G. D. 2012 Experimental study of the role of the Weber and capillary numbers on Mesler entrainment. AIChE J. 58, 3841-3851.

Scheid, B., Dorbolo, S., Arriaga, L. R. \& Rio, E. 2012 Antibubble dynamics: the drainage of an air film with viscous interfaces. Phys. Rev. Lett. 109, 264502. 
Scheid, B., Zawala, J. \& Dorbolo, S. 2014 Gas dissolution in antibubble dynamics. Soft Matt. 10, 7096-7102.

Sigler, J. \& Mesler, R. 1990 The behavior of the gas film formed upon drop impact with a liquid surface. J. Colloid Interface Sci. 134 (2), 459-474.

Sundberg-Anderson, R. K. \& SAYlor, J. R. 2014 Mesler entrainment in alcohols. Exp. Fluids 55, 1653.

Thoroddsen, S. T., Етон, T. G. \& Takehara, K. 2003 Air entrapment under an impacting drop. J. Fluid Mech. 478, 125-134.

Thoroddsen, S. T., Etoh, T. G. \& Takehara, K. 2007 Experiments on bubble pinch-off. Phys. Fluids 19, 042101.

Thoroddsen, S. T., Thoraval, M.-J., Takehara, K. \& Etoh, T. G. 2012 Micro-bubble morphologies following drop impacts onto a pool surface. J. Fluid Mech. 708, 469-479.

TомотіKA, S. 1936 Breaking up of a drop of viscous liquid immersed in another viscous fluid which is extending at a uniform rate. Proc. R. Soc. Lond. A 153, 302-318.

Tran, T., De Maleprade, H., Sun, C. \& Lohse, D. 2013 Air entrainment during impact of droplets on liquid surfaces. J. Fluid Mech. 726, R3.

Vandewalle, N., Terwagne, D., Gilet, T., Caps, H. \& Dorbolo, S. 2009 Antibubbles, liquid onions and bouncing droplets. Colloids Surf. A 344, 42-47. 